This is a self-archived version of an original article. This version may differ from the original in pagination and typographic details.

Author(s): Guo, Chang-Yu; Hencl, Stanislav; Tengvall, Ville

Title: Mappings of finite distortion : size of the branch set

Year: 2020

Version: Accepted version (Final draft)

Copyright: (c) 2018 Walter de Gruyter GmbH, Berlin/Boston

Rights: $C C$ BY-NC-ND 4.0

Rights url: https://creativecommons.org/licenses/by-nc-nd/4.0/

Please cite the original version:

Guo, C.-Y., Hencl, S., \& Tengvall, V. (2020). Mappings of finite distortion : size of the branch set. Advances in Calculus of Variations, 13(4), 325-360. https://doi.org/10.1515/acv-2017-0034 


\title{
MAPPINGS OF FINITE DISTORTION: SIZE OF THE BRANCH SET
}

\author{
CHANG-YU GUO, STANISLAV HENCL, AND VILLE TENGVALL
}

\begin{abstract}
We study the branch set of a mapping between subsets of $\mathbb{R}^{n}$, i.e., the set where a given mapping is not defining a local homeomorphism. We construct several sharp examples showing that the branch set or its image can have positive measure.
\end{abstract}

\section{INTRODUCTION}

Let $\Omega \subset \mathbb{R}^{n}, n \geq 2$, be a domain and let us consider a continuous mapping $f: \Omega \rightarrow \mathbb{R}^{n}$. In many applications it is important to know when this mapping is invertible (see e.g. [5] and [33]) or at least locally invertible in many places (see e.g. [31], [12]) as the invertibility is the important key tool for the study of topological properties of the mapping. The usual proof of invertibility is to first show that the mapping is discrete (preimage $f^{-1}(y)$ of every point $y \in \mathbb{R}^{n}$ is a discrete set, i.e., does not have an accumulation point in $\Omega$ ) and open (image of each open set is an open set), as each discrete and open mapping is invertible in many points as we mention below (see e.g. [21], [12], [22] and [16] for results in this direction). Moreover, openness and discreteness together with reasonable conditions around $\partial \Omega$ imply that the mapping is invertible.

However, every continuous, discrete and open mapping $f: \Omega \rightarrow \mathbb{R}^{n}$ is not necessarily injective or invertible in a neighborhood of each point in $\Omega$. The simplest example is the winding mapping $\omega: \mathbb{R}^{n} \rightarrow \mathbb{R}^{n}$ defined as

$$
\omega\left(r \cos (\theta), r \sin (\theta), x_{3}, \ldots, x_{n}\right)=\left(r \cos (2 \theta), r \sin (2 \theta), x_{3}, \ldots, x_{n}\right),
$$

where $r=\sqrt{x_{1}^{2}+x_{2}^{2}} \geq 0$ and $\theta \in \mathbb{R}$ for $x=\left(x_{1}, x_{2}, \ldots, x_{n}\right) \in \mathbb{R}^{n}$. This mapping will wind the space twice around the $(n-2)$-dimensional hyperplane $H_{n-2}:=\{(0,0)\} \times$ $\mathbb{R}^{n-2}$ and therefore it is not defining a local homeomorphism in a neighborhood of any point which lies in $H_{n-2}$. This kind of behavior of a mapping where the injectivity is destroyed is usually called branching and the set of points where a mapping $f$ is not defining a local homeomorphism is called the branch set of $f$. We will denote the branch set and its image of a mapping $f$ by $\mathcal{B}_{f}$ and $f\left(\mathcal{B}_{f}\right)$, respectively. These sets have very rich and complex topological and geometrical structures both of which have been studied in a variety of different contexts, see e.g. [1, 2, 12, 13, 14, 25, 35] and references there.

Date: March 29, 2018.

2010 Mathematics Subject Classification. 30C65,26B10.

Key words and phrases. Mappings of finite distortion, branch set, Lusin's condition $(N)$.

C.Y. Guo was supported by Swiss National Science Foundation Grant 153599 and 165848. S. Hencl and V. Tengvall were supported by the ERC CZ grant LL1203 of the Czech Ministry of Education. V.T. was also supported by the Academy of Finland Project 277923. 
In the case of the winding mapping we see that even if there is some branching occurring it is only happening in a very small set. Namely, the branch set $\mathcal{B}_{\omega}$ and its image $\omega\left(\mathcal{B}_{\omega}\right)$ both have topological and Hausdorff dimensions equal to $n-2$ and so have Lebesgue $n$-measure zero. By the theorem of Chernavskiu $[7,8]$, see also the work by Väisälä [35] for a shorter proof, for the topological dimension of these sets we have

$$
\operatorname{dim}_{\mathcal{T}} \mathcal{B}_{f}=\operatorname{dim}_{\mathcal{T}} f\left(\mathcal{B}_{f}\right) \leq n-2
$$

for every continuous, discrete and open mapping $f$. Therefore, the size of the branch set and its image in the sense of the topological dimension are the maximal ones for the winding map. Moreover, in the case $n=2$ the branch set and its image for continuous, discrete and open mapping contain only isolated points and hence (1.1) holds in this case also for the Hausdorff dimension. However, when $n \geq 3$ there are no topological obstacles to make the branch set very large with respect to the (Lebesgue) measure and Hausdorff dimension. To see this, we simply choose a homeomorphism $g: \mathbb{R}^{n} \rightarrow \mathbb{R}^{n}$ which sends a given Cantor set of positive measure to a subset of the hyperplane $H_{n-2}$ (see e.g. [10]) and then define

$$
f:=g^{-1} \circ \omega \circ g,
$$

which gives us a continuous, discrete and open mapping with both sets $\mathcal{B}_{f}$ and $f\left(\mathcal{B}_{f}\right)$ having positive measure. Therefore, we need to have some additional assumptions in order to control the measure of the branch set and its image for continuous, discrete and open mappings. In this paper these additional assumptions will be given in terms of mappings of finite distortion as it is natural in many applications (see e.g. $[21,22,16,19,15])$ :

Definition 1.1 (Mapping of finite distortion). Let $\Omega \subset \mathbb{R}^{n}$ be a domain with $n \geq 2$. We say that a mapping $f \in W_{\mathrm{loc}}^{1,1}\left(\Omega, \mathbb{R}^{n}\right)$ is a mapping of finite distortion if $(F D-1) J_{f} \in L_{\text {loc }}^{1}(\Omega)$,

(FD-2) $J_{f}(x)>0$ a.e. on the set where $|D f(x)|>0$.

For each such a mapping we associate an outer distortion function $K_{O}(\cdot, f): \Omega \rightarrow$ $[1, \infty]$ and an inner distortion function $K_{I}(\cdot, f): \Omega \rightarrow[1, \infty]$ defined as

$$
K_{O}(x, f)=\left\{\begin{array}{ll}
\frac{|D f(x)|^{n}}{J_{f}(x)}, & \text { if } J_{f}(x)>0 \\
1, & \text { otherwise }
\end{array} \quad \text { and } \quad K_{I}(x, f)= \begin{cases}\frac{\left|D^{\sharp} f(x)\right|^{n}}{J_{f}(x)^{n-1}}, & \text { if } J_{f}(x)>0 \\
1, & \text { otherwise. }\end{cases}\right.
$$

Above, and in what follows $J_{f}(x):=\operatorname{det} D f(x)$ stands for the pointwise Jacobian and $D^{\sharp} f(x)$ for the pointwise adjugate matrix of the differential matrix $D f(x)$. We will refer by $|A|$ the operator norm of a matrix $A$. Moreover, we point out that for the distortion functions described above we have the following useful pointwise inequalities

$$
K_{I}(x, f) \leq K_{O}^{n-1}(x, f) \quad \text { and } \quad K_{O}(x, f) \leq K_{I}^{n-1}(x, f)
$$

for almost every $x \in \Omega$, see e.g. [19]. For the readers who are not familiar with mappings of finite distortion we recommend the monographs $[4,15,19]$.

In order to study the size of the branch set and its image for mappings of finite distortion we first recall that when $K_{O}(\cdot, f) \in L^{\infty}(\Omega)$ in Definition 1.1 we recover 
the well studied class of mappings of bounded distortion, also known as quasiregular mappings. Furthermore, if a mapping of bounded distortion is injective we call it quasiconformal. By the fundamental theorem of Reshetnyak (see e.g. [31]) every nonconstant mapping of bounded distortion is continuous, discrete and open. Moreover (see e.g. [32]), nonconstant mappings of bounded distortion

(1) are differentiable almost everywhere,

(2) have positive Jacobian almost everywhere, and

(3) satisfy Lusin's conditions $(N)$ (i.e. every set of measure zero is mapped to a set of measure zero).

The properties listed above play an important role in the study of the size of the branch set and its image. Indeed, by [32, Lemma I.4.11] every continuous, discrete and open Sobolev mapping which is differentiable almost everywhere and has almost everywhere nonvanishing Jacobian determinant defines a local homeomorphism in a neighborhood of almost every point. In addition, if the mapping satisfies Lusin's condition $(N)$ then the image of the branch set will have measure zero as well. In particular, it follows that both the branch set of a nonconstant mapping of bounded distortion and its image are of measure zero. Therefore, it is natural to ask if this is the case even when the boundedness of the distortion is relaxed. The first result of this paper gives sharp conformality conditions under which continuous, discrete and open mappings of finite distortion may have branch set of positive measure. We give these conditions in terms of the inner distortion function:

Theorem 1.2. Let $n \geq 3$ and $\Omega \subset \mathbb{R}^{n}$ be open. Then we have the following:

(i) Suppose $f \in W_{\text {loc }}^{1, n-1}\left(\Omega, \mathbb{R}^{n}\right)$ is a continuous, discrete and open mapping of finite distortion with

$$
K_{I}(\cdot, f) \in L_{\mathrm{loc}}^{1}(\Omega) .
$$

Then $f$ is an almost everywhere differentiable mapping with positive Jacobian determinant almost everywhere. Especially, we have $\mathcal{L}^{n}\left(\mathcal{B}_{f}\right)=0$.

(ii) On the other hand, there exists a continuous, discrete and open Lipschitz mapping $f \in W_{\mathrm{loc}}^{1, \infty}\left(\mathbb{R}^{n}, \mathbb{R}^{n}\right)$ of finite distortion with

$$
K_{I}(\cdot, f) \in L_{\text {loc }}^{p}\left(\mathbb{R}^{n}\right)
$$

for every $0<p<1$ such that $\mathcal{L}^{n}\left(\mathcal{B}_{f}\right)>0$.

We should point out that the conformality and Sobolev assumptions in Theorem 1.2 are too weak to guarantee continuity, discreteness and openness of the mapping, see e.g. [16]. Because of this we will assume these conditions, in addition, to stay in the right class of mappings. The positive statement in Theorem 1.2 follows directly from the results in $[25,34]$. Moreover, the local integrability assumption of the inner distortion function is sharp for the differentiability almost everywhere even in the class of $W^{1, n-1}$-homeomorphisms of finite distortion, see [17]. It is well-known that this is also the right integrability class for the inner distortion function to guarantee that the zero set of the Jacobian has null measure.

In some sense it is surprising that after we lose local integrability of the inner distortion we may find, not only $W^{1, n-1}$-mappings, but even Lipschitz mappings with the branch set of positive measure. Especially, we may see that differentiability almost everywhere is not a sufficient condition for the local invertibility almost everywhere as 
every Lipschitz mapping is differentiable at almost every point. In addition, Theorem 1.2 shows the necessity of nonvanishing Jacobian almost everywhere for the local invertibility. Thus, we may ask if this is also a sufficient condition for this property. Notice that by [25] every continuous, discrete and open mapping of finite distortion with positive Jacobian almost everywhere satisfies Lusin's condition $\left(N^{-1}\right)$, i.e., the set $f^{-1}(E)$ has measure zero for every set $E \subset \mathbb{R}^{n}$ of measure zero. Therefore, to construct a continuous, discrete and open mapping of finite distortion with a positive Jacobian almost everywhere and with the branch set of positive measure it is necessary to have the measure of the image of the branch positive as well. In the main result of this paper we will construct such a mapping showing that the condition $J_{f}>0$ is not sufficient for the local invertibility:

Theorem 1.3. Let $n=3$. There exists a continuous, discrete and open mapping of finite distortion such that $f \in W^{1, p}\left((-1,1)^{3},(-1,1)^{3}\right)$ for all $p \in[1,2), J_{f}>0$ almost everywhere, but

$$
\mathcal{L}^{n}\left(\mathcal{B}_{f}\right)>0 \quad \text { and } \quad \mathcal{L}^{n}\left(f\left(\mathcal{B}_{f}\right)\right)>0 .
$$

We would like to remark that the mapping $f$ as in Theorem 1.3 cannot be differentiable almost everywhere. Recall that by the differentiability result of Väisälä [37] every continuous and open mapping in $W^{1, p}, p>n-1$, is differentiable almost everywhere, and on the other hand differentiability might fail for mappings in $W^{1, n-1}$ even if they are assumed to be homeomorphisms of finite distortion with positive Jacobian almost everywhere, see e.g. [9]. Hence, it would be interesting to know what happens for the size of the branch set in Theorem 1.3 in the borderline case where $f \in W^{1, n-1}$ as it could also reveal if the differentiability almost everywhere is a necessary condition for the local invertibility. It is possible to slightly improve the Sobolev regularity of the mapping in Theorem 1.3 on logarithmic scale, and to derive some level of integrability for the distortion functions of this mapping, see section 7 . However, we do not know what are the sharp Lorentz conditions and distortion assumptions under which a continuous, discrete and open mapping of finite distortion with positive Jacobian almost everywhere will have branch set of zero measure. For the sharp Lorentz condition for the differentiability without any additional conformality conditions we refer to [29].

There are several reasons to study the branch set of mappings of finite distortion. One such a reason can be seen when we try to generalize the well-known Poletsky's [30] and Väisälä's [36] moduli inequalities of quasiregular mappings, or the corresponding capacity inequalities, see e.g. [27, 28, 35], for mappings of finite distortion. These inequalities were generalized for mappings of finite distortion in $W_{\text {loc }}^{1, n}$ with locally integrable inner distortion by Koskela and Onninen [26]. The optimal assumptions for these inequalities with lower regularity assumptions were further studied in [11, 17, 34]. As it was observed in [11], these inequalities would follow for a continuous, discrete and open mapping $f$ of finite distortion with even lower regularity assumptions by following the standard arguments in the theory of quasiregular mappings whenever

(1) the mapping $f$ is differentiable almost everywhere,

(2) the Jacobian determinant is positive almost everywhere,

(3) the branch set $\mathcal{B}_{f}$ has measure zero, and 
(4) the image $f\left(\mathcal{B}_{f}\right)$ has measure zero as well.

It follows from $[25,34]$ that the conditions (1)-(3) hold whenever we have a continuous, discrete and open mapping $f \in W_{\mathrm{loc}}^{1, n-1}\left(\Omega, \mathbb{R}^{n}\right)$ of finite distortion with locally integrable inner distortion. However, we need to have much stronger assumptions if we want (4) to hold:

Theorem 1.4. Let $\Omega \subset \mathbb{R}^{n}$ be an open set with $n \geq 3$ and let $\lambda>0$. Then we have the following:

(i) If $f: \Omega \rightarrow \mathbb{R}^{n}$ is a nonconstant mapping of finite distortion with

$$
\exp \left(\lambda K_{O}(\cdot, f)\right) \in L_{\mathrm{loc}}^{1}(\Omega),
$$

then $f$ is continuous, discrete and open. Moreover, $f$ satisfies Lusin's condition $(N)$ and $\mathcal{L}^{n}\left(f\left(\mathcal{B}_{f}\right)\right)=0$.

(ii) On the other hand, for each $0<\varepsilon<1$ there is a continuous, discrete and open mapping $f \in W_{\mathrm{loc}}^{1, p}\left(\mathbb{R}^{n}, \mathbb{R}^{n}\right)$, for all $1 \leq p<n$, of finite distortion such that

$$
\exp \left(K_{O}^{1-\varepsilon}(\cdot, f)\right) \in L_{\mathrm{loc}}^{1}\left(\mathbb{R}^{n}\right)
$$

and $\mathcal{L}^{n}\left(f\left(\mathcal{B}_{f}\right)\right)>0$.

Theorem 1.4 shows the necessity of Lusin's condition $(N)$ for the condition $\mathcal{L}^{n}\left(f\left(\mathcal{B}_{f}\right)\right)=$ 0 . We will see in section 3 that Theorem 1.4 (ii) follows from an even stronger result, Theorem 3.2. For the positive statement in Theorem 1.4, see [18, 22, 23, 24]. For the reader interested in the positive statement in the planar case, we also refer to [20].

\section{PRELIMINARIES}

2.1. Notation. A point $x \in \mathbb{R}^{n}$ in coordinates is denoted as $\left(x_{1}, x_{2}, \ldots, x_{n}\right)$ and its Euclidean and supremum norms are denoted by $|x|:=\sqrt{\sum_{i=1}^{n} x_{i}^{2}}$ and $\|x\|:=\sup _{i}\left|x_{i}\right|$. Furthermore, we will denote by

$$
Q(a, r):=\left(a_{1}-r, a_{1}+r\right) \times \cdots \times\left(a_{n}-r, a_{n}+r\right)
$$

an open cube centered at $a \in \mathbb{R}^{n}$ with edge length $2 r>0$, i.e., the set $\left\{x \in \mathbb{R}^{n}\right.$ : $\|x-a\|<r\}$. The interior of a set $A \subset \mathbb{R}^{n}$ is denoted by $A^{\circ}$ and the symbol $\mathcal{L}^{n}$ refers to the Lebesgue $n$-measure. When we write

$$
C:=C\left(p_{1}, \ldots, p_{k}\right)
$$

we mean a positive constant which depends only on the given parameters $p_{1}, \ldots p_{k}$. The constant $C$ might change from line to line. Furthermore, for given functions $f$ and $g$ we denote $f \lesssim g$ if there exists a positive constant $C>0$ such that $f(x) \leq C g(x)$ for almost every $x$. In the case when both conditions $f \lesssim g$ and $g \lesssim f$ are satisfied we denote $f \sim g$.

2.2. Sobolev spaces. Let $\Omega \subset \mathbb{R}^{n}$ be an open set. The Sobolev space $W^{1, p}\left(\Omega, \mathbb{R}^{m}\right)$, $1 \leq p \leq \infty$, will consist of all the mappings

$$
f:=\left(f_{1}, \ldots, f_{m}\right): \Omega \rightarrow \mathbb{R}^{m}
$$


such that for the real-valued coordinate functions and for their distributional partial derivatives we have

$$
f_{i} \in L^{p}(\Omega) \quad \text { and } \quad \frac{\partial f_{i}}{\partial x_{j}} \in L^{p}(\Omega)
$$

for all $i=1, \ldots, m$ and for every $j=1, \ldots, n$. If $f \in W^{1, p}\left(\Omega^{\prime}, \mathbb{R}^{m}\right)$ for every subdomain $\Omega^{\prime} \subset \subset \Omega$ we denote $f \in W_{\text {loc }}^{1, p}\left(\Omega, \mathbb{R}^{m}\right)$.

2.3. ACL condition. Let $i \in\{1,2, \ldots, n\}$ and denote by $\pi_{i}$ the projection on the given hyperplane $H_{i}=\left\{x \in \mathbb{R}^{m}: x_{i}=0\right\}$ perpendicular to the $x_{i}$-axis. We say that a mapping $f \in L_{\mathrm{loc}}^{1}\left(\Omega, \mathbb{R}^{m}\right)$ is absolutely continuous (on lines) if the following ACL condition holds:

(ACL) For every cube $Q(a, r)=\left(a_{1}-r, a_{1}+r\right) \times \cdots \times\left(a_{n}-r, a_{n}+r\right) \subset \subset \Omega$ and for every $i \in\{1,2, \ldots, n\}$ the coordinate functions

$$
f^{i}(t ; x):=f\left(x_{1}, \ldots, x_{i-1}, t, x_{i+1}, \ldots, x_{n}\right)
$$

are absolutely continuous (with variable $t$ ) on $\left(a_{i}-r, a_{i}+r\right)$ for $\mathcal{L}^{n-1}$-almost every $x \in \pi_{i}(Q(a, r))$.

The following characterization of Sobolev spaces is classical and can be found e.g. in [3, Section 3.11] and [15, Theorem A.15]:

Proposition 2.1. Let $\Omega \subset \mathbb{R}^{n}$ be an open set and $f \in L_{\mathrm{loc}}^{p}\left(\Omega, \mathbb{R}^{m}\right)$ for some $1 \leq p<$ $\infty$. Then $f \in W_{\text {loc }}^{1, p}\left(\Omega, \mathbb{R}^{m}\right)$ if and only if there is a representative of $f$ which is an $\operatorname{ACL}\left(\Omega, \mathbb{R}^{m}\right)$ mapping with locally $L^{p}$-integrable partial derivatives on $\Omega$.

2.4. Conditions $(N)$ and $\left(N^{-1}\right)$, and the area formula. Suppose $f: \Omega \rightarrow \mathbb{R}^{n}$ is a mapping defined on an open set $\Omega \subset \mathbb{R}^{n}$ with $n \geq 1$.

(1) We say that $f$ satisfies Lusin's condition $(N)$ if for each set $E \subset \Omega$ such that $\mathcal{L}^{n}(E)=0$ we have $\mathcal{L}^{n}(f(E))=0$.

(2) We say that $f$ satisfies Lusin's condition $\left(N^{-1}\right)$ if for each set $F \subset \mathbb{R}^{n}$ such that $\mathcal{L}^{n}(F)=0$ we have $\mathcal{L}^{n}\left(f^{-1}(F)\right)=0$.

We will need the following well-known area formula for Sobolev mappings (see e.g. $[15$, Theorem A.12]):

Proposition 2.2. Let $f \in W_{\text {loc }}^{1,1}\left(\Omega, \mathbb{R}^{n}\right)$ and let $\eta$ be a nonnegative Borel measurable function on $\mathbb{R}^{n}$. Then

$$
\int_{\Omega} \eta(f(x))|J(x, f)| \mathrm{d} x \leq \int_{\mathbb{R}^{n}} \eta(y) N(y, f, \Omega) \mathrm{d} y,
$$

where the multiplicity function $N(y, f, \Omega)$ is defined as the number of points in the set $f^{-1}(y) \cap \Omega$ for $y \in \mathbb{R}^{n}$. Moreover, there is an equality in (2.1) if we assume in addition that $f$ satisfies Lusin's condition $(N)$.

2.5. Constructing Cantor sets. Suppose $[-1,1]^{n} \subset \mathbb{R}^{n}$, and denote by $\mathbb{V}$ the set of $2^{n}$ vertices of the cube $[-1,1]^{n}$. The sets

$$
\mathbb{V}^{k}=\mathbb{V} \times \cdots \times \mathbb{V}, \quad k \in \mathbb{N}
$$

will serve as the set of indices for our construction. 
Next, suppose $\left\{a_{k}\right\}_{k=0}^{\infty}$ is a decreasing sequence such that $1=a_{0} \geq a_{1} \geq \cdots>0$, and define

$$
r_{k}=2^{-k} a_{k} .
$$

Set $z_{0}=0$. Then it follows that $Q\left(z_{0}, r_{0}\right)=(-1,1)^{n}$ and we proceed further by induction. For $\boldsymbol{v}(k)=\left(v_{1}, \ldots, v_{k}\right) \in \mathbb{V}^{k}$ we denote $\boldsymbol{w}(k)=\left(v_{1}, \ldots, v_{k-1}\right)$ and define (see Fig. 1)

$$
\begin{aligned}
& z_{\boldsymbol{v}(k)}=z_{\boldsymbol{w}(k)}+\frac{1}{2} r_{k-1} v_{k}=z_{0}+\frac{1}{2} \sum_{j=1}^{k} r_{j-1} v_{j}, \\
& Q_{\boldsymbol{v}(k)}^{\prime}=Q\left(z_{\boldsymbol{v}(k)}, 2^{-k} a_{k-1}\right) \text { and } Q_{\boldsymbol{v}(k)}=Q\left(z_{\boldsymbol{v}(k)}, 2^{-k} a_{k}\right) .
\end{aligned}
$$

Then for the measure of the $k$-th frame $Q_{\boldsymbol{v}(k)}^{\prime} \backslash Q_{\boldsymbol{v}(k)}$ we have

$$
\mathcal{L}^{n}\left(Q_{\boldsymbol{v}(k)}^{\prime} \backslash Q_{\boldsymbol{v}(k)}\right)=2^{-k n}\left(a_{k-1}^{n}-a_{k}^{n}\right) .
$$

Formally we should write $\boldsymbol{w}(\boldsymbol{v}(k))$ instead of $\boldsymbol{w}(k)$ but for the simplification of the notation we will avoid this.
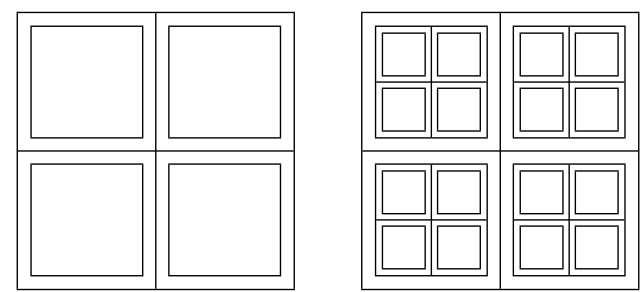

Fig. 1 Cubes $Q_{\boldsymbol{v}(k)}$ and $Q_{\boldsymbol{v}(k)}^{\prime}$ for $k=1,2$.

It is not difficult to find out that the resulting Cantor set

$$
\bigcap_{k=1}^{\infty} \bigcup_{\boldsymbol{v}(k) \in \mathbb{V}^{k}} Q_{\boldsymbol{v}(k)}=: C\left[\left\{a_{k}\right\}_{k=0}^{\infty}\right]=\mathcal{C}_{a} \times \cdots \times \mathcal{C}_{a}
$$

is a product of $n$ Cantor sets $\mathcal{C}_{a}$ in $\mathbb{R}$, and the number of the cubes $\left\{Q_{\boldsymbol{v}(k)}: \boldsymbol{v}(k) \in \mathbb{V}^{k}\right\}$ is $2^{n k}$. Therefore, the measure of the Cantor set $C_{A}:=C\left[\left\{a_{k}\right\}_{k}\right]$ can be calculated as

$$
\mathcal{L}^{n}\left(C_{A}\right)=\lim _{k \rightarrow \infty} 2^{n k}\left(2 a_{k} 2^{-k}\right)^{n}=\lim _{k \rightarrow \infty} 2^{n} a_{k}^{n} .
$$

2.6. Homeomorphism that maps a Cantor set onto another one. Let us first recall [15, Lemma 2.1].

Lemma 2.3. Let $\rho:(0, \infty) \rightarrow(0, \infty)$ be a strictly monotone function and $\rho \in$ $C^{1}((0, \infty))$. Then for the mapping

$$
f(x)=\frac{x}{\|x\|} \rho(\|x\|), \quad x \neq 0
$$

we have for almost every $x$

$$
|D f(x)| \sim \max \left\{\frac{\rho(\|x\|)}{\|x\|},\left|\rho^{\prime}(\|x\|)\right|\right\} \text { and } J_{f}(x) \sim \rho^{\prime}(\|x\|)\left(\frac{\rho(\|x\|)}{\|x\|}\right)^{n-1} .
$$


In fact, this lemma is shown in [15] for the Euclidean norm $|x|$ but by a bi-Lipschitz change of variables it it easy to see that the same formula holds also for the supremum norm $\|x\|=\max _{i=1, \ldots, n}\left|x_{i}\right|$ analogously to [15, Chapter 4.3].

Suppose

$$
C_{A}:=C\left[\left\{a_{k}\right\}_{k=0}^{\infty}\right] \quad \text { and } \quad C_{B}:=C\left[\left\{b_{k}\right\}_{k=0}^{\infty}\right]
$$

are two Cantor sets which we get by applying the algorithm in section 2.5 to the sequences $\left\{a_{k}\right\}_{k=0}^{\infty}$ and $\left\{b_{k}\right\}_{k=0}^{\infty}$. Following section 2.5 we define

$$
\begin{aligned}
& r_{k}=2^{-k} a_{k}, \quad r_{k}^{\prime}=2^{-k} a_{k-1}, \quad \tilde{r}_{k}=2^{-k} b_{k}, \quad \tilde{r}_{k}^{\prime}=2^{-k} b_{k-1}, \\
& z_{\boldsymbol{v}(k)}=z_{\boldsymbol{w}(k)}+\frac{1}{2} r_{k-1} v_{k}=z_{0}+\frac{1}{2} \sum_{j=1}^{k} r_{j-1} v_{j} \quad \text { and } \\
& \tilde{z}_{\boldsymbol{v}(k)}=\tilde{z}_{\boldsymbol{w}(k)}+\frac{1}{2} \tilde{r}_{k-1} v_{k}=\tilde{z}_{0}+\frac{1}{2} \sum_{j=1}^{k} \tilde{r}_{j-1} v_{j} .
\end{aligned}
$$

Furthermore, we also define

$$
\begin{array}{ll}
Q_{\boldsymbol{v}(k)}^{\prime}=Q\left(z_{\boldsymbol{v}(k)}, r_{k}^{\prime}\right), & Q_{\boldsymbol{v}(k)}=Q\left(z_{\boldsymbol{v}(k)}, r_{k}\right) \\
\tilde{Q}_{\boldsymbol{v}(k)}^{\prime}=Q\left(\tilde{z}_{\boldsymbol{v}(k)}, \tilde{r}_{k}^{\prime}\right), & \tilde{Q}_{\boldsymbol{v}(k)}=Q\left(\tilde{z}_{\boldsymbol{v}(k)}, \tilde{r}_{k}\right) .
\end{array}
$$

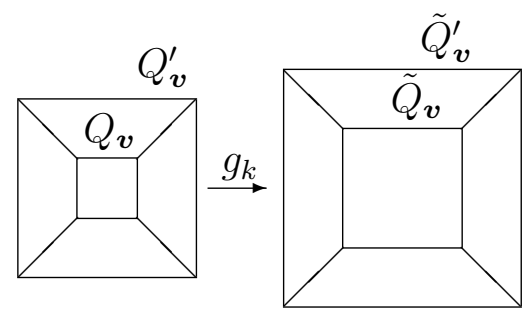

Fig. 2 The transformation of $Q_{\boldsymbol{v}}^{\prime} \backslash Q_{\boldsymbol{v}}^{\circ}$ onto $\tilde{Q}_{\boldsymbol{v}}^{\prime} \backslash \tilde{Q}_{\boldsymbol{v}}^{\circ}$

It remains to find a homeomorphism $g$ which maps $C_{A}$ onto $C_{B}$. We will find a sequence of homeomorphisms $g_{k}:(-1,1)^{n} \rightarrow(-1,1)^{n}$. Set $g_{0}(x)=x$ and we proceed by induction. We will give a mapping $g_{1}$ which stretches each cube $Q_{\boldsymbol{v}}, \boldsymbol{v} \in \mathbb{V}^{1}$, homogeneously so that $g_{1}\left(Q_{\boldsymbol{v}}\right)$ equals $\tilde{Q}_{\boldsymbol{v}}$. On the annulus $Q_{\boldsymbol{v}}^{\prime} \backslash Q_{\boldsymbol{v}}, g_{1}$ is defined to be an appropriate radial map (with respect to the supremum norm) with respect to $z_{\boldsymbol{v}}$ and $\tilde{z}_{\boldsymbol{v}}$ in the image in order to make $g_{1}$ a homeomorphism. The general step is the following: If $k>1, g_{k}$ is defined as $g_{k-1}$ outside the union of all cubes $Q_{\boldsymbol{w}}$, $\boldsymbol{w} \in \mathbb{V}^{k-1}$. Further, $g_{k}$ remains equal to $g_{k-1}$ at the centers of cubes $Q_{\boldsymbol{v}}, \boldsymbol{v} \in \mathbb{V}^{k}$. Then $g_{k}$ stretches each cube $Q_{\boldsymbol{v}}, \boldsymbol{v} \in \mathbb{V}^{k}$, homogeneously so that $g_{k}\left(Q_{\boldsymbol{v}}\right)$ equals $\tilde{Q}_{\boldsymbol{v}}$. On the annulus $Q_{\boldsymbol{v}}^{\prime} \backslash Q_{\boldsymbol{v}}, g_{k}$ is defined to be an appropriate radial map (with respect to the supremum norm) with respect to $z_{\boldsymbol{v}}$ in the preimage and $\tilde{z}_{\boldsymbol{v}}$ in the image to make $g_{k}$ a homeomorphism (see Fig. 2). Notice that the Jacobian determinant $J_{g_{k}}(x)$ will be strictly positive almost everywhere in $(-1,1)^{n}$. 
In this construction we use the notation $\|x\|$ for the supremum norm of $x \in \mathbb{R}^{n}$. The mappings $g_{k}, k \in \mathbb{N}$, are formally defined as

$$
g_{k}(x)= \begin{cases}g_{k-1}(x) & \text { for } x \notin \bigcup_{\boldsymbol{v} \in \mathbb{V}^{k}} Q_{\boldsymbol{v}}^{\prime} \\ g_{k-1}\left(z_{\boldsymbol{v}}\right)+\left(\alpha_{k}\left\|x-z_{\boldsymbol{v}}\right\|+\beta_{k}\right) \frac{x-z_{\boldsymbol{v}}}{\left\|x-z_{\boldsymbol{v}}\right\|} & \text { for } x \in Q_{\boldsymbol{v}}^{\prime} \backslash Q_{\boldsymbol{v}}, \boldsymbol{v} \in \mathbb{V}^{k} \\ g_{k-1}\left(z_{\boldsymbol{v}}\right)+\frac{\tilde{r}_{k}}{r_{k}}\left(x-z_{\boldsymbol{v}}\right) & \text { for } x \in Q_{\boldsymbol{v}}, \boldsymbol{v} \in \mathbb{V}^{k}\end{cases}
$$

where the constants $\alpha_{k}$ and $\beta_{k}$ are given by

$$
\alpha_{k} r_{k}+\beta_{k}=\tilde{r}_{k} \text { and } \alpha_{k} \frac{r_{k-1}}{2}+\beta_{k}=\frac{\tilde{r}_{k-1}}{2} \text {. }
$$

It is not difficult to find out that each $g_{k}$ is a homeomorphism and maps

$$
\bigcup_{\boldsymbol{v} \in \mathbb{V}^{k}} Q_{\boldsymbol{v}} \text { onto } \bigcup_{\boldsymbol{v} \in \mathbb{V}^{k}} \tilde{Q}_{\boldsymbol{v}}
$$

The mapping $g(x)=\lim _{k \rightarrow \infty} g_{k}(x)$ is clearly one to one and continuous and therefore it is a homeomorphism. Moreover, it is not difficult to see that $g$ is differentiable almost everywhere, absolutely continuous on almost all lines parallel to coordinate axes and maps $C_{A}$ onto $C_{B}$ (see [15, Chapter 4.3] for details).

Let $k \in \mathbb{N}$ and $\boldsymbol{v} \in \mathbb{V}^{k}$. We need to estimate $D g(x)$ in the interior of the annulus $Q_{\boldsymbol{v}}^{\prime} \backslash Q_{\boldsymbol{v}}$. Since

$$
g(x)=g\left(z_{\boldsymbol{v}}\right)+\left(\alpha_{k}\left\|x-z_{\boldsymbol{v}}\right\|+\beta_{k}\right) \frac{x-z_{\boldsymbol{v}}}{\left\|x-z_{\boldsymbol{v}}\right\|}
$$

we obtain from Lemma 2.3 and (2.5) that

$$
D g(x) \sim \max \left\{\frac{\tilde{r}_{k}}{r_{k}}, \alpha_{k}\right\}=\max \left\{\frac{\tilde{r}_{k}}{r_{k}}, \frac{\frac{\tilde{r}_{k-1}}{2}-\tilde{r}_{k}}{\frac{r_{k-1}}{2}-r_{k}}\right\}
$$

and

$$
J_{g}(x) \sim \alpha_{k}\left(\frac{\tilde{r}_{k}}{r_{k}}\right)^{n-1}=\frac{\frac{\tilde{r}_{k-1}}{2}-\tilde{r}_{k}}{\frac{r_{k-1}}{2}-r_{k}}\left(\frac{\tilde{r}_{k}}{r_{k}}\right)^{n-1} .
$$

2.7. Constructing Cantor's tower. Suppose $n \geq 2$ and denote by $\hat{\mathbb{V}}$ the set of points

$$
\left(0,0, \ldots, 0,-1+\frac{2 j}{2^{n}}\right)
$$

where $j=1,2, \ldots, 2^{n}$. The sets

$$
\hat{\mathbb{V}}^{k}:=\hat{\mathbb{V}} \times \cdots \times \hat{\mathbb{V}}, \quad k \in \mathbb{N},
$$

will serve as the set of indices in the construction of Cantor's tower.

Suppose next $\left\{c_{k}\right\}_{k=0}^{\infty}$ is a decreasing sequence such that $1=c_{0} \geq c_{1} \geq c_{2} \geq \cdots>0$, and define

$$
\hat{r}_{k}:=2^{-n k} c_{k} .
$$

Set $\hat{z}_{0}=0$. Then it follows that $Q\left(\hat{z}_{0}, \hat{r}_{0}\right)=(-1,1)^{n}$ and we proceed further by induction. For $\hat{\boldsymbol{v}}(k):=\left(\hat{v}_{1}, \hat{v}_{2}, \ldots, \hat{v}_{k}\right) \in \hat{\mathbb{V}}^{k}$ we denote $\hat{\boldsymbol{w}}(k):=\left(\hat{v}_{1}, \hat{v}_{2}, \ldots, \hat{v}_{k-1}\right)$ and 
define (see Fig. 3)

$$
\begin{aligned}
& \hat{z}_{\boldsymbol{v}(k)}:=\hat{z}_{\hat{\boldsymbol{w}}(k)}+\hat{r}_{k-1} \hat{v}_{k}=\hat{z}_{0}+\sum_{j=1}^{k} \hat{r}_{j-1} \hat{v}_{j} \\
& \hat{Q}_{\boldsymbol{v}(k)}^{\prime}:=Q\left(\hat{z}_{\hat{\boldsymbol{v}}(k)}, 2^{-n k} c_{k-1}\right) \text { and } \hat{Q}_{\boldsymbol{v}(k)}:=Q\left(\hat{z}_{\hat{\boldsymbol{v}}(k)}, 2^{-n k} c_{k}\right)
\end{aligned}
$$
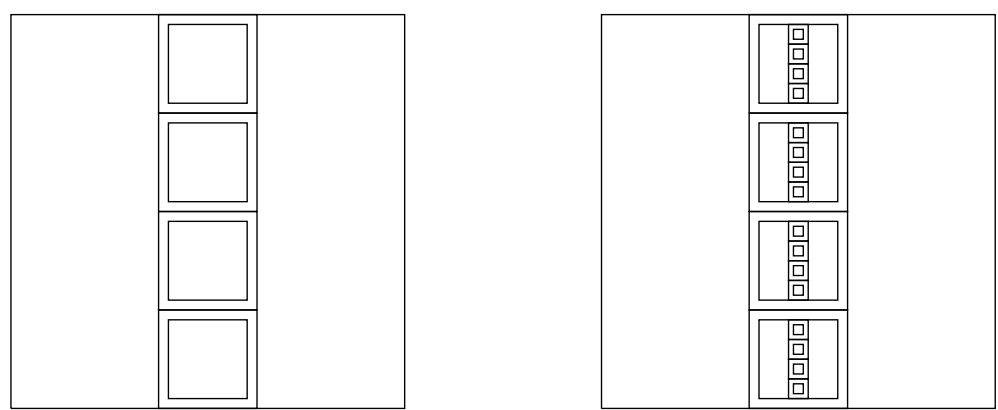

Fig. 3 Cubes $\hat{Q}_{\boldsymbol{v}(k)}$ and $\hat{Q}_{\boldsymbol{v}(k)}^{\prime}$ for $k=1,2$ in the construction of the Cantor's tower.

2.8. Bi-Lipschitz mapping which takes a Cantor set onto a Cantor's tower. Let us now define the Cantor set $C_{B}$ in section 2.5 by choosing

$$
b_{k}=2^{-k \beta},
$$

where $\beta \geq n+1$. Similarly, we define the Cantor's tower $C_{C}^{T}$ in section 2.7 by choosing

$$
c_{k}=2^{-k \beta} \text {. }
$$

As $\beta \geq n+1$, we see that

$$
\hat{Q}_{\boldsymbol{v}(k)}=Q\left(\hat{z}_{\hat{\boldsymbol{v}}(k)}, 2^{-k n} c_{k}\right) \subset Q\left(\hat{z}_{\hat{\boldsymbol{v}}(k)}, 2^{-1-n k} c_{k-1}\right)=\frac{1}{2} \hat{Q}_{\boldsymbol{v}(k)}^{\prime}
$$

and thus we have enough empty space in $\hat{Q}_{\boldsymbol{v}(k)}^{\prime} \backslash \hat{Q}_{\boldsymbol{v}(k)}$ to move the cubes of the next generation into a tower formation.

The following proposition will give us a bi-Lipschitz mapping $F: \mathbb{R}^{n} \rightarrow \mathbb{R}^{n}$ which maps the Cantor set $C_{B}$ onto Cantor's tower $C_{B}^{T}$. We refer to this mapping as a tower mapping and it is applied to prove theorems 1.2 and 1.4. Later we need to refine this mapping further in order to prove Theorem 1.3.

Proposition 2.4. Suppose that $C_{B}$ is the Cantor set and $C_{B}^{T}$ the Cantor's tower in $\mathbb{R}^{n}$ defined by the sequence

$$
b_{k}=2^{-k \beta},
$$

where $\beta \geq n+1$. Then there is a bi-Lipschitz mapping $L: \mathbb{R}^{n} \rightarrow \mathbb{R}^{n}$ which takes $C_{B}$ onto $C_{B}^{T}$.

Proof. We construct bi-Lipschitz mappings $L_{j}$ which take the $j$-th step of the construction of $C_{B}$ onto the $j$-th step of the construction of $C_{B}^{T}$. All $L_{j}$ are bi-Lipschitz with the same constant and the $\operatorname{limit} F=\lim _{j \rightarrow \infty} L_{j}$ is thus bi-Lipschitz as well. 


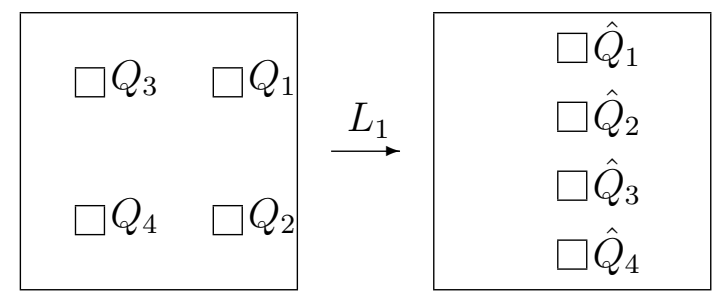

Fig. 4 Construction of $L_{1}$ which translates $Q_{i}$ onto $\hat{Q}_{i}$.

We claim that there is a bi-Lipschitz mapping $L_{1}:[-1,1]^{n} \rightarrow[-1,1]^{n}$ such that (see Fig. 4)

$$
\begin{aligned}
& L_{1}(x)=x \text { for every } x \in \partial[-1,1]^{n} \text { and } \\
& L_{1}(x)=x-z_{\boldsymbol{v}(1)}+\hat{z}_{\boldsymbol{v}(1)} \text { for every } x \in Q_{\boldsymbol{v}(1)} .
\end{aligned}
$$

This means that $L_{1}$ fixes the boundary and it is a just a translation of each of the $2^{n}$ cubes $Q_{\boldsymbol{v}(1)}$ onto $\hat{Q}_{\boldsymbol{v}(1)}$. As $\beta \geq n+1$ we have enough space for doing this (see (2.8)) and it is not difficult to see that such a homeomorphism exists (see Fig. 4) and it can be constructed as a bi-Lipschitz mapping. Formally we should give the exact formula for the mapping which would not be difficult but it would be extremely lengthy. We skip this as the construction of such $F_{1}$ is trustworthy and not difficult to see from the picture. More details and the construction of admissible $F_{1}$ for $n=3$ can be found in Section 7 . In fact the construction there is slightly different as we map cubes $Q_{\boldsymbol{v}(1)}$ onto $\hat{Q}_{\boldsymbol{v}(1)}$ but the mapping is not necessarily just a translation there.

We continue by constructing $L_{2}$ as a scaled copy of $L_{1}$ into each $Q_{\boldsymbol{v}(1)}$. Formally, we set

$$
L_{2}(x)= \begin{cases}L_{1}(x) & \text { for } x \in[-1,1]^{n} \backslash \bigcup_{\boldsymbol{v}(1) \in \mathbb{V}^{1}} Q_{\boldsymbol{v}(1)}, \\ \hat{z}_{\boldsymbol{v}(1)}+2^{-1-\beta} L_{1}\left(2^{1+\beta}\left(x-z_{\boldsymbol{v}(1)}\right)\right) & \text { for } x \in Q_{\boldsymbol{v}(1)} .\end{cases}
$$

As the constructions of $C_{B}$ and $C_{B}^{T}$ are self-similar it is not difficult to see that $F_{2}$ translates each $Q_{\boldsymbol{v}(2)}$ onto the corresponding $\hat{Q}_{\boldsymbol{v}(2)}$. We claim that $L_{2}$ has the same bi-Lipschitz constant $L>0$ as $L_{1}$. For $x, y \in Q_{\boldsymbol{v}(1)}$ we can estimate

$$
\begin{aligned}
\left|L_{2}(x)-L_{2}(y)\right| & \leq 2^{-1-\beta} \mid L_{1}\left(2^{1+\beta}\left(x-z_{\boldsymbol{v}(1)}\right)-L_{1}\left(2^{1+\beta}\left(y-z_{\boldsymbol{v}(1)}\right) \mid\right.\right. \\
& \leq 2^{-1-\beta} L 2^{1+\beta}|x-y|=L|x-y|
\end{aligned}
$$

and a similar estimate holds from below. If $x \in Q_{\boldsymbol{v}(1)}$ and $y \notin Q_{\boldsymbol{v}(1)}$ we can connect $x$ and $y$ by a segment, divide this segment by points on the boundaries of $\bigcup_{\boldsymbol{v}(1) \in \mathbb{V}^{1}} Q_{\boldsymbol{v}(1)}$, use the estimate on each part of the segment separately and sum up. Thus $F_{2}$ is also $L$-bi-Lipschitz.

We continue by induction and we set

$$
L_{k+1}(x)= \begin{cases}L_{k}(x) & \text { for } x \in[-1,1]^{n} \backslash \bigcup_{\boldsymbol{v}(k) \in \mathbb{V} k} Q_{\boldsymbol{v}(k)}, \\ \hat{z}_{\boldsymbol{v}(k)}+2^{(-1-\beta) k} L_{1}\left(2^{k(1+\beta)}\left(x-z_{\boldsymbol{v}(k)}\right)\right) & \text { for } x \in Q_{\boldsymbol{v}(k)} .\end{cases}
$$

Similarly as for $L_{2}$ we obtain that $L_{k+1}$ is $L$-bi-Lipschitz and it maps each $Q_{\boldsymbol{v}(k+1)}$ onto the corresponding $\hat{Q}_{\boldsymbol{v}(k+1)}$ affinely. The limiting map $F(x)=\lim _{j \rightarrow \infty} L_{j}(x)$ is thus $L$-bi-Lipschitz and maps $C_{B}$ onto $C_{B}^{T}$. 


\section{Proof of Theorem 1.4: the Size of $f\left(\mathcal{B}_{f}\right)$}

We have already noted in the introduction that if $f$ has "reasonably nice" properties then the set $f\left(\mathcal{B}_{f}\right)$ has measure zero. The next lemma specifies these reasonably nice properties.

Lemma 3.1. Suppose $f$ is a continuous, sense-preserving, discrete and open mapping which is differentiable almost everywhere and satisfies Lusin's condition $(N)$. Then $\mathcal{L}^{n}\left(f\left(\mathcal{B}_{f}\right)\right)=0$.

Proof. Since the issue is local, we may assume that $f$ has bounded multiplicity [32, Proposition I.4.10 (3)]. Let us denote

$$
\begin{aligned}
& A:=\left\{x \in \mathcal{B}_{f}: f \text { is not differentiable at } x\right\} \text { and } \\
& B:=\left\{x \in \mathcal{B}_{f}: f \text { is differentiable at } x\right\} .
\end{aligned}
$$

Then we may write $\mathcal{B}_{f}=A \cup B$. Because $f$ is differentiable almost everywhere we have $\mathcal{L}^{n}(A)=0$ and hence it follows from Lusin's condition $(N)$ that

$$
\mathcal{L}^{n}(f(A))=0 .
$$

On the other hand, by [32, Lemma I.4.11] we have $J_{f}(x)=0$ for every $x \in B$, and thus the area formula (see Proposition 2.2) implies

$$
\int_{f(B)} N(y, f, f(B)) \mathrm{d} x=\int_{B}\left|J_{f}\right|(x) \mathrm{d} x=0 .
$$

As $N(y, f, f(B)) \geq 1$ on $f(B)$ we obtain that $\mathcal{L}^{n}(f(B))=0$ and it follows from (3.1) that

$$
\mathcal{L}^{n}\left(f\left(\mathcal{B}_{f}\right)\right) \leq \mathcal{L}^{n}(f(A))+\mathcal{L}^{n}(f(B))=0
$$

The following theorem stresses the importance of Lusin's condition $(N)$. Namely, without the condition $(N)$ we cannot assure that the set $f\left(\mathcal{B}_{f}\right)$ has measure zero even when the branch set $\mathcal{B}_{f}$ has measure zero.

Theorem 3.2. Let $\Psi:(0, \infty) \rightarrow(0, \infty)$ be a strictly increasing continuous function such that

$$
\int_{1}^{\infty} \frac{\Psi^{\prime}(t)}{t} \mathrm{~d} t<\infty
$$

Then there exists a discrete and open mapping $f: \mathbb{R}^{n} \rightarrow \mathbb{R}^{n}, n \geq 3$, with the following properties:

1) $f \in W_{\text {loc }}^{1, p}\left(\mathbb{R}^{n}, \mathbb{R}^{n}\right)$ for all $1 \leq p<n$,

2) $f$ is a mapping of finite distortion, and the outer distortion $K_{O}(\cdot, f)$ satisfies

$$
\exp \left(\Psi\left(K_{O}(x, f)\right)\right) \in L_{\text {loc }}^{1}\left(\mathbb{R}^{n}\right),
$$

3) the inner distortion $K_{I}(\cdot, f)$ satisfies

$$
\exp \left(\Psi^{*}\left(K_{I}(x, f)\right)\right) \in L_{\mathrm{loc}}^{1}\left(\mathbb{R}^{n}\right),
$$

where $\Psi^{*}(t)=\Psi\left(t^{1 /(n-1)}\right)$,

4) the mapping $f$ is differentiable almost everywhere, the Jacobian of $f$ is strictly positive almost everywhere and $f$ satisfies Lusin's condition $\left(N^{-1}\right)$, and 
5) $\mathcal{L}^{n}\left(\mathcal{B}_{f}\right)=0$, but $\mathcal{L}^{n}\left(f\left(\mathcal{B}_{f}\right)\right)>0$.

Proof. Suppose that $\omega: \mathbb{R}^{n} \rightarrow \mathbb{R}^{n}$ is the winding mapping defined as in the introduction. Then $\omega$ is a quasiregular mapping with the branch set $\mathcal{B}_{\omega}=\{(0,0)\} \times \mathbb{R}^{n-2}$.

Let us define Cantor sets $C_{A}:=C\left[\left\{a_{k}\right\}_{k=0}^{\infty}\right]$ and $C_{B}:=C\left[\left\{b_{k}\right\}_{k=0}^{\infty}\right]$ (see section 2.5) by setting

$$
a_{k}:=\frac{1}{2}\left(1+2^{-2 k}\right) \quad \text { and } \quad b_{k}=2^{-2 k}
$$

Then it follows that

$$
\mathcal{L}^{n}\left(C_{A}\right)>1 \text { and } \quad \mathcal{L}^{n}\left(C_{B}\right)=0 .
$$

Let $g: \mathbb{R}^{n} \rightarrow \mathbb{R}^{n}$ be a bi-Lipschitz mapping (obtained as the inverse of the tower mapping from section 2.8) such that $C_{B} \subset g\left(\{0\}^{n-1} \times[-1,1]\right)$, where $\{0\}^{n-1}$ stands for the origin in $\mathbb{R}^{n-1}$.

Suppose next $\Psi$ and $\Psi^{*}$ are defined as in the theorem. Then by [23, Corollary C] there exists a homeomorphism $F:[-1,1]^{n} \rightarrow[-1,1]^{n}$ that fixes the boundary $\partial Q(0,1)$ and has the following properties:

(a) $F \in W^{1,1}\left((-1,1)^{n}, \mathbb{R}^{n}\right), F$ is differentiable almost everywhere, and

$$
F \in W^{1, q}\left((-1,1)^{n}, \mathbb{R}^{n}\right) \text { for every } 1 \leq q<n .
$$

This follows from $|D F(x)|^{n} \leq K_{O}(x, F) J_{F}(x), J_{F} \in L^{1}$ and $K_{O}(x, F) \in L^{a}$ for every $a<\infty$.

(b) The Jacobian determinant $J_{F}(x)$ is positive almost everywhere, and

$$
\int_{(-1,1)^{n}} J_{F}(x) \mathrm{d} x<\infty .
$$

(c) The distortion $K_{O}(x, F)$ is finite almost everywhere, and for every $\lambda>0$

$$
\int_{(-1,1)^{n}} \exp \left(\lambda \Psi\left(K_{O}(x, F)\right)\right) \mathrm{d} x<\infty
$$

where $\Psi$ is given as in the statement (2) of the Theorem 3.2.

(d) The mapping $F$ maps a Cantor set $C_{B}$ of zero measure onto a Cantor set $C_{A}$ of positive measure.

All the properties listed above remain valid locally for $\hat{F}: \mathbb{R}^{n} \rightarrow \mathbb{R}^{n}$ defined by

$$
\hat{F}(x)= \begin{cases}F(x), & \text { if } x \in[-1,1]^{n} \\ x, & \text { if } x \in \mathbb{R}^{n} \backslash[-1,1]^{n} .\end{cases}
$$

Finally, we define $f: \mathbb{R}^{n} \rightarrow \mathbb{R}^{n}$ by

$$
f:=\hat{F} \circ g \circ \omega .
$$

Then it is easy to see that $f$ is a continuous, discrete and open mapping such that

$$
f\left(\mathcal{B}_{f}\right)=\hat{F} \circ g\left((0,0) \times \mathbb{R}^{n-2}\right) \supset \hat{F}\left(C_{B}\right)=C_{A} .
$$

Therefore it suffices to show that $f$ has finite distortion and satisfies the conditions (1)-(4): 
Condition (1): Set $h:=F \circ g$. Since $g$ is bi-Lipschitz, $h$ has the same regularity as $F$. For the winding mapping $\omega$, it is locally bi-Lipschitz outside the set $A:=$ $\{(0,0)\} \times \mathbb{R}^{n-2}$ of zero measure, and thus we know that $f$ is ACL and that the chain rule applies outside the set $A$. Note also that $J_{\omega}(x)=2=|D \omega(x)|$ for all $x$. Suppose now that $E \subset \mathbb{R}^{n}$ is a bounded open set. Then by applying the chain rule on the set $E \backslash A$ we get

$$
\begin{aligned}
\int_{E}|D f|^{p} \mathrm{~d} x & =\int_{E \backslash A}|D f|^{p} \mathrm{~d} x \leq \int_{E \backslash A}|D(h \circ \omega)|^{p}|D \omega|^{p} \mathrm{~d} x \\
& =\int_{E \backslash A}|D h(\omega(x))|^{p} J_{\omega}(x) \frac{|D \omega(x)|^{p}}{J_{\omega}(x)} \mathrm{d} x \\
& =2^{p-1} \int_{E \backslash A}|D h(\omega(x))|^{p} J_{\omega}(x) \mathrm{d} x \\
& \leq 2^{p} \int_{\omega(E \backslash A)}|D h(x)|^{p} \mathrm{~d} x<\infty,
\end{aligned}
$$

which implies that $f \in W_{\text {loc }}^{1, p}\left(\mathbb{R}^{n}, \mathbb{R}^{n}\right)$.

Condition (2): We show next that $f$ has finite distortion. Firstly by applying the chain rule we find out that

$$
D f(x)=D h(\omega(x)) D \omega(x) \quad \text { and } \quad J_{f}(x)=J_{h}(\omega(x)) J_{\omega}(x)
$$

for all $x \in \mathbb{R}^{n} \backslash A$. Since $\omega$ is a quasiregular mapping, it satisfies Lusin's condition $\left(N^{-1}\right)$ and it follows from (3.3) and from the fact that $h=F \circ g$ is a mapping of finite distortion that $J_{f}(x)>0$ almost everywhere in the set $|D f(x)|>0$. Moreover,

$$
\int_{E} J_{f}=\int_{E \backslash A} J_{f}=\int_{E \backslash A} J_{h}(\omega(x)) J_{\omega}(x) \mathrm{d} x \leq 2 \int_{h(E \backslash A)} J_{h}<\infty .
$$

Thus, $f$ is a mapping of finite distortion.

Moreover, we have

$$
\begin{aligned}
\int_{U} \exp \left(\Psi\left(K_{O}(x, f)\right)\right) \mathrm{d} x & \leq \int_{U} \exp \left(\Psi\left(K_{O}(\omega(x), h) K_{\omega}\right)\right) \frac{J_{\omega}(x)}{J_{\omega}(x)} \mathrm{d} x \\
& \leq \int_{U} \exp \left(\Psi\left(2^{n-1} K_{O}(\omega(x), h)\right)\right) J_{\omega}(x) \mathrm{d} x \\
& \leq 2 \int_{\omega(U)} \exp \left(\Psi\left(2^{n-1} K_{O}(y, h)\right)\right) \mathrm{d} y<\infty,
\end{aligned}
$$

for every $U \subset \subset \mathbb{R}^{n}$.

Condition (3): Next, set $\Psi^{*}(t):=\Psi\left(t^{1 /(n-1)}\right)$. Then by the pointwise inequality $K_{I}(x, f) \leq K_{O}(x, f)^{n-1}$ and by Condition (2) we get

$$
\int_{U} \exp \left(\Psi^{*}\left(K_{I}(x, f)\right)\right) \mathrm{d} x \leq \int_{U} \exp \left(\Psi\left(K_{O}(x, f)\right)\right) \mathrm{d} x<\infty,
$$

as desired. 
Condition (4): We know that $\omega$ is differentiable almost everywhere, $h$ is differentiable almost everywhere and that $\omega$ satisfies Lusin $\left(N^{-1}\right)$ condition. It thus follows from (3.3) that $f$ is differentiable almost everywhere. Similarly we obtain from (3.3) that $J_{f}>0$ almost everywhere as this holds for both $\omega$ and $h$.

Condition (5): Now we observe that $\mathcal{B}_{f}=\{(0,0)\} \times \mathbb{R}^{n-2}$ and thus

$$
\mathcal{L}^{n}\left(\mathcal{B}_{f}\right)=\mathcal{L}^{n}\left(\{(0,0)\} \times \mathbb{R}^{n-2}\right)=0 .
$$

On the other hand, it holds that $f\left(\mathcal{B}_{f}\right) \supset C_{A}$, and therefore

$$
\mathcal{L}^{n}\left(f\left(\mathcal{B}_{f}\right)\right) \geq \mathcal{L}^{n}\left(C_{A}\right)>0
$$

which ends the proof.

Proof of Theorem 1.4. It is well-known that assumptions of $(i)$ imply that $f$ is continuous, sense-preserving, discrete and open, differentiable almost everywhere and satisfies Lusin's condition $(N)$, see [18, 22, 23, 24]. It follows from Lemma 3.1 that $\mathcal{L}^{n}\left(f\left(\mathcal{B}_{f}\right)\right)=0$.

The statement of $(i i)$ follows from Theorem 3.2 if we choose $\Psi(t)=\frac{t}{\log ^{1+\varepsilon}(e+t)}$ for $t$ large enough.

\section{Proof of Theorem 1.2: The Size of $\mathcal{B}_{f}$}

Proof of Theorem 1.2. As we have already pointed out, if a continuous, discrete and open mapping is both

(1) differentiable almost everywhere, and

(2) has non-vanishing Jacobian almost everywhere

then the branch set of the mapping has measure zero (see [32, Lemma I.4.11]). The claim of $(i)$ follows as we know from $[25]$ that $K_{I}(\cdot, f) \in L_{\text {loc }}^{1}(\Omega)$ implies $J_{f}>0$ almost everywhere and from [34] we know that every continuous, discrete and open mapping of finite distortion $f \in W_{\text {loc }}^{1, n-1}\left(\Omega, \mathbb{R}^{n}\right)$ with locally integrable inner distortion function is differentiable almost everywhere.

To prove part $(i i)$ let us define Cantor sets $C_{A}:=C\left[\left\{a_{k}\right\}_{k=0}^{\infty}\right]$ and $C_{B}:=C\left[\left\{b_{k}\right\}_{k=0}^{\infty}\right]$ by setting

$$
a_{k}:=\frac{1}{2}\left(1+2^{-k \beta}\right) \quad \text { and } \quad b_{k}=2^{-k \beta},
$$

where $\beta \geq n+1$ (see Section 2.8 for such choice of $\beta$ ). Then it follows that

$$
\mathcal{L}^{n}\left(C_{A}\right)=1 \text { and } \mathcal{L}^{n}\left(C_{B}\right)=0 .
$$

Suppose that $F$ is the homeomorphism which maps $C_{A}$ onto $C_{B}$ and takes each annulus $Q_{\boldsymbol{v}}^{\prime} \backslash Q_{\boldsymbol{v}}$ radially onto the corresponding annulus $\tilde{Q}_{\boldsymbol{v}}^{\prime} \backslash \tilde{Q}_{\boldsymbol{v}}$ (see section 2.6 for details). Then in the interior of each annulus $Q_{\boldsymbol{v}}^{\prime} \backslash Q_{\boldsymbol{v}}$ we get by applying (2.6) and (2.7) that

$$
|D F(x)| \sim \max \left\{\frac{b_{k}}{a_{k}}, \frac{b_{k-1}-b_{k}}{a_{k-1}-a_{k}}\right\} \sim \max \left\{2^{-k \beta}, 1\right\}=1 \quad \text { and } \quad\left|D^{\sharp} F(x)\right| \sim 2^{-k \beta(n-2)} .
$$

In addition, we also have

$$
J_{F}(x) \sim\left(\frac{b_{k}}{a_{k}}\right)^{n-1} \frac{b_{k-1}-b_{k}}{a_{k-1}-a_{k}} \sim\left(2^{-k \beta}\right)^{n-1} .
$$


Hence

$$
K_{O}(x, F)=\frac{|D f(x)|^{n}}{J_{f}(x)} \sim\left(2^{k \beta}\right)^{n-1} \quad \text { and } \quad K_{I}(x, F)=\frac{\left|D^{\sharp} f(x)\right|^{n}}{J_{f}(x)^{n-1}} \sim 2^{k \beta} .
$$

It follows that $F$ is Lipschitz and if $0<p<\frac{1}{n-1}$ then

$$
\begin{aligned}
\int_{Q_{0}} K_{O}(x, F)^{p} \mathrm{~d} x & =\int_{C_{A}} K_{O}(x, F)^{p} \mathrm{~d} x+\int_{Q_{0} \backslash C_{A}} K_{O}(x, F)^{p} \mathrm{~d} x \\
& \leq \mathcal{L}^{n}\left(C_{A}\right)+C \sum_{k=1}^{\infty} 2^{k n} 2^{-k n} a_{k}^{n-1}\left(a_{k}-a_{k+1}\right)\left(2^{k \beta}\right)^{(n-1) p} \\
& \leq \mathcal{L}^{n}\left(C_{A}\right)+C \sum_{k=1}^{\infty} 2^{k n} 2^{-k n} 1^{n-1} 2^{-k \beta}\left(2^{k \beta}\right)^{(n-1) p}<\infty
\end{aligned}
$$

and similarly for every $0<q<1$ we get

$$
\begin{aligned}
\int_{Q_{0}} K_{I}(x, F)^{q} \mathrm{~d} x & =\int_{C_{A}} K_{I}(x, F)^{q} \mathrm{~d} x+\int_{Q_{0} \backslash C_{A}} K_{I}(x, F)^{q} \mathrm{~d} x \\
& \leq \mathcal{L}^{n}\left(C_{A}\right)+C \sum_{k=1}^{\infty} 2^{k n} 2^{-k n} a_{k}^{n-1}\left(a_{k}-a_{k+1}\right)\left(2^{k \beta}\right)^{q} \\
& \leq \mathcal{L}^{n}\left(C_{A}\right)+C \sum_{k=1}^{\infty} 2^{k n} 2^{-k n} 1^{n-1} 2^{-k \beta}\left(2^{k \beta}\right)^{q}<\infty
\end{aligned}
$$

Above we have used the fact that $K_{O}(x, f)=K_{I}(x, f)=1$ for almost every $x \in C_{A}$.

Suppose next that $g: \mathbb{R}^{n} \rightarrow \mathbb{R}^{n}$ is the bi-Lipschitz mapping (defined in section 2.8) which takes the Cantor set $C_{B}$ onto the Cantor's tower $C_{B}^{T}$. Furthermore, let $\omega: \mathbb{R}^{n} \rightarrow \mathbb{R}^{n}$ be the winding map. Then both mappings $g$ and $\omega$ are quasiregular and therefore there exist constants $K_{g} \geq 1$ and $K_{\omega} \geq 1$ such that

$$
|D g(x)|^{n} \leq K_{g} J_{g}(x) \text { and }|D \omega(x)|^{n} \leq K_{\omega} J_{\omega}(x)
$$

for almost every $x$. We also have

$$
\left|D^{\sharp} g(x)\right|^{n} \leq K_{g}^{n-1} J_{g}(x)^{n-1} \quad \text { and } \quad\left|D^{\sharp} \omega(x)\right|^{n} \leq K_{\omega}^{n-1} J_{\omega}(x)^{n-1}
$$

for almost every $x$.

Now we define the mapping $f: \mathbb{R}^{n} \rightarrow \mathbb{R}^{n}$ as

$$
f:=\omega \circ g \circ F \text {. }
$$

Then $F$ maps $C_{A}$ onto $C_{B}$ which is mapped by $g$ into the segment $\{0\}^{n-1} \times[-1,1]$. Since $\omega$ is winding around this segment, all points on this segment belong to the branch set. The branch set of $f$ thus contains the whole set $C_{A}$ and we get $\mathcal{L}^{n}\left(\mathcal{B}_{f}\right)>0$.

Furthermore, $f$ is a Lipschitz mapping as a composition of three Lipschitz mappings. It is easy to see that $|D F(x)|=0$ and $J_{F}(x)=0$ for almost every $x \in C_{A}$ and the composition with Lipschitz mapping cannot change this. Hence $|D f(x)|=0$ and $J_{f}(x)=0$ for almost every $x \in C_{A}$ and consequently

$$
K_{O}(x, f)=1 \quad \text { for almost every } x \in C_{A} .
$$


With a similar way we may also show that

$$
K_{I}(x, f)=1 \quad \text { for almost every } x \in C_{A} .
$$

Moreover, $F$ is locally bi-Lipschitz outside of $C_{A}$ and hence preimages of sets (outside of $C_{A}$ ) of zero measure have zero measure. It follows that $g$ has classical derivative at $F(x)$ for almost every $x \notin C_{A}$ (as the preimage under $F$ of points of non-differentiability of $g$ has zero measure). As $g$ is bi-Lipschitz we can argue similarly to deduce that the derivative of $\omega$ exists at a point $g(F(x))$ for almost every $x \notin C_{A}$. By the chain rule we obtain that for almost every $x \notin C_{A}$ we have

$$
\begin{gathered}
|D f(x)|=|D \omega(g(F(x)))||D g(F(x))||D F(x)|, \text { and } \\
\left|D^{\sharp} f(x)\right|=\left|D^{\sharp} \omega(g(F(x)))\right|\left|D^{\sharp} g(F(x))\right|\left|D^{\sharp} F(x)\right| .
\end{gathered}
$$

In addition, we also get

$$
J_{f}(x)=J_{\omega}(g(F(x))) J_{g}(F(x)) J_{F}(x) .
$$

It follows from (4.3)-(4.9) that

$$
\begin{aligned}
& K_{O}(x, f) \leq \frac{|D f(x)|^{n}}{J_{f}(x)} \leq K_{\omega} K_{g} \frac{|D F(x)|^{n}}{J_{F}(x)} \text {, and } \\
& K_{I}(x, f) \leq \frac{\left|D^{\sharp} f(x)\right|^{n}}{J_{f}(x)^{n-1}} \leq K_{\omega}^{n-1} K_{g}^{n-1} \frac{\left|D^{\sharp} F(x)\right|^{n}}{J_{F}(x)^{n-1}}
\end{aligned}
$$

for almost every $x \in Q_{0} \backslash C_{A}$. Finally, by (4.5), (4.10) and (4.1) we conclude $K_{O}(\cdot, f) \in$ $L_{\text {loc }}^{p}\left(Q_{0}\right)$ for all $0<p<\frac{1}{n-1}$. Similarly, by (4.6), (4.10) and (4.2) we conclude that $K_{I}(\cdot, f) \in L_{\mathrm{loc}}^{q}\left(Q_{0}\right)$ for all $0<q<1$.

\section{A mapping Which maps a Cantor set onto a Cantor set and CORNERS ONTO CORNERS}

5.1. Strategy of the proof. Before the proof of Theorem 1.3 we explain the overall strategy. We start with a naive approach of how to construct a counterexample and explain why it fails. Let us start with a mapping $g$ (as in section 2.6) which maps a Cantor type set $C_{A}$ of positive measure onto a Cantor type set $C_{B}$ of zero measure and assume that these Cantor sets are defined using sequences

$$
a_{k}=\frac{1}{2}\left(1+\frac{1}{2^{k}}\right) \text { and } b_{k}=2^{-k \beta} \text { where } \beta \text { is big enough. }
$$

Then we apply the mapping $L$ (defined in subsection 2.8) which takes a Cantor type set of zero measure onto a Cantor's tower. After this we apply the winding mapping $\omega$ and then we return the mapping back, i.e., we have

$$
f=g^{-1} \circ L^{-1} \circ \omega \circ L \circ g .
$$

It is clear that both $\mathcal{B}_{f}$ and $f\left(\mathcal{B}_{f}\right)$ contain the Cantor type set of positive measure $C_{A}$. However, this mapping $f$ does not belong to $W^{1,1}$. Indeed, let us estimate its derivative. As before we have on the $k$-th frame by (2.6) that

$$
|D g| \sim \max \left\{2^{-\beta k}, 2^{k-\beta k}\right\} \text { and }\left|D g^{-1}\right| \sim \max \left\{2^{\beta k}, 2^{\beta k-k}\right\}
$$


and the derivatives of $L, \omega$, and $L^{-1}$ are bounded as these mapping are Lipchitz. Hence we can estimate

$$
|D f| \leq C 2^{\beta k} 2^{k-\beta k}=C 2^{k}
$$

there as in general the "radial" and "nonradial" part of the derivative of $D g$ and $D g^{-1}$ can meet as the Lipschitz mappings between them change the directions. The measure of the $k$-frame is comparable to $2^{-k}$ and hence

$$
\int|D f| \leq C \sum_{k=1}^{\infty} 2^{-k} 2^{k}=\infty
$$

The last sum will diverge for any change of parameters in the construction of the Cantor sets.

It follows that we have to do something more subtle. We divide the $k$-th frame into corners (=parts close to the edges) and non-corners - see Fig. 16. The measure of the corners in the construction of $C_{A}$ is comparable to $2^{-2 k}$. In this section we construct an analogy of the mapping $g$ that maps $C_{A}$ onto $C_{B}$ while mapping corners of $C_{A}$ (quite a small set) onto corners of $C_{B}$ (quite a big set). In the next section we construct an analogy of the bi-Lipschitz tower mapping in a more delicate manner and we control the position of the image of the corners. In fact, we can achieve that there is a sector of a small but fixed angle $\theta$ (see Fig. 17) around the central line of the rotation which is inside the image of the corners. Instead of the usual winding mapping $\omega$ we consider a sectorial winding $\omega_{\theta}$ which winds the sector with an opening angle $\theta$ once aroud the $z$-axis in such a way that the angle $\theta$ is mapped to angle $2 \pi+\theta$. In addition, we define $\omega_{\theta}$ as the identity map outside the sector. This $\omega_{\theta}$ is still a local bi-Lipschitz map outside the $z$-axis and now our mapping

$$
g^{-1} \circ L^{-1} \circ \omega_{\theta} \circ L \circ g
$$

is identity outside of the corners and the big derivative can happen only inside the union of the corners. As the measure of corners is bounded by $C 2^{-2 k}$ we obtain

$$
\int|D f|^{p} \leq C \sum_{k=1}^{\infty} 2^{-2 k} 2^{p k}<\infty \text { for every } 1 \leq p<2 .
$$

5.2. Mapping corners onto corners. To prove Theorem 1.3 we need to construct a special homeomorphism which maps a Cantor set of positive measure onto a Cantor set of zero measure in such a way that the corners of the first construction are mapped close to the corners of the second construction. Here by corners we mean those parts of the annulus $Q_{\boldsymbol{v}(k)}^{\prime} \backslash Q_{\boldsymbol{v}(k)}$ that are close to the edges and vertices (see Fig. 16 in section 5.5), the formal definition is given later. As we pointed out in section 5.1, the standard construction (see the mapping $g$ in section 2.6) is not good for us as the (relatively small) corners of the first Cantor construction are not mapped onto the (relatively big) corners of the second Cantor construction. We have to define the mapping close to the vertices so that it is stretching much more there.

Let us define the Cantor sets $C_{A}:=C\left[\left\{a_{k}\right\}_{k=0}^{\infty}\right]$ and $C_{B}:=C\left[\left\{b_{k}\right\}_{k=0}^{\infty}\right]$ by setting

$$
a_{k}=\frac{1}{2}\left(1+\frac{1}{2^{k}}\right) \text { and } \quad b_{k}=2^{-k \beta},
$$


where $\beta \geq n+1$. Then

$$
\mathcal{L}^{n}\left(C_{A}\right)=\lim _{k \rightarrow \infty} 2^{n k}\left(2 a_{k} 2^{-k}\right)^{n}=\lim _{k \rightarrow \infty}\left(1+\frac{1}{2^{k}}\right)^{n}=1
$$

and

$$
\mathcal{L}^{n}\left(C_{B}\right)=\lim _{k \rightarrow \infty} 2^{n k}\left(2 b_{k} 2^{-k}\right)^{n}=\lim _{k \rightarrow \infty} 2^{-k n \beta}=0
$$

5.3. Planar case. For simplicity and comprehensibility we give the details of the construction first for $n=2$ and then we generalize this construction to the case $n=3$. Set

$$
r_{k}=2^{-k} a_{k} \quad \text { and } \quad \tilde{r}_{k}=2^{-k} b_{k}
$$

and recall that the cubes in the definition of the Cantor sets $C_{A}$ and $C_{B}$ (see section 2.5) are defined as

$$
Q_{\boldsymbol{v}(k)}^{\prime}=Q\left(z_{\boldsymbol{v}(k)}, \frac{r_{k-1}}{2}\right) \quad \text { and } \quad Q_{\boldsymbol{v}(k)}=Q\left(z_{\boldsymbol{v}(k)}, r_{k}\right)
$$

and

$$
\tilde{Q}_{\boldsymbol{v}(k)}^{\prime}=Q\left(\tilde{z}_{\boldsymbol{v}(k)}, \frac{\tilde{r}_{k-1}}{2}\right) \quad \text { and } \quad \tilde{Q}_{\boldsymbol{v}(k)}=Q\left(\tilde{z}_{\boldsymbol{v}(k)}, \tilde{r}_{k}\right)
$$
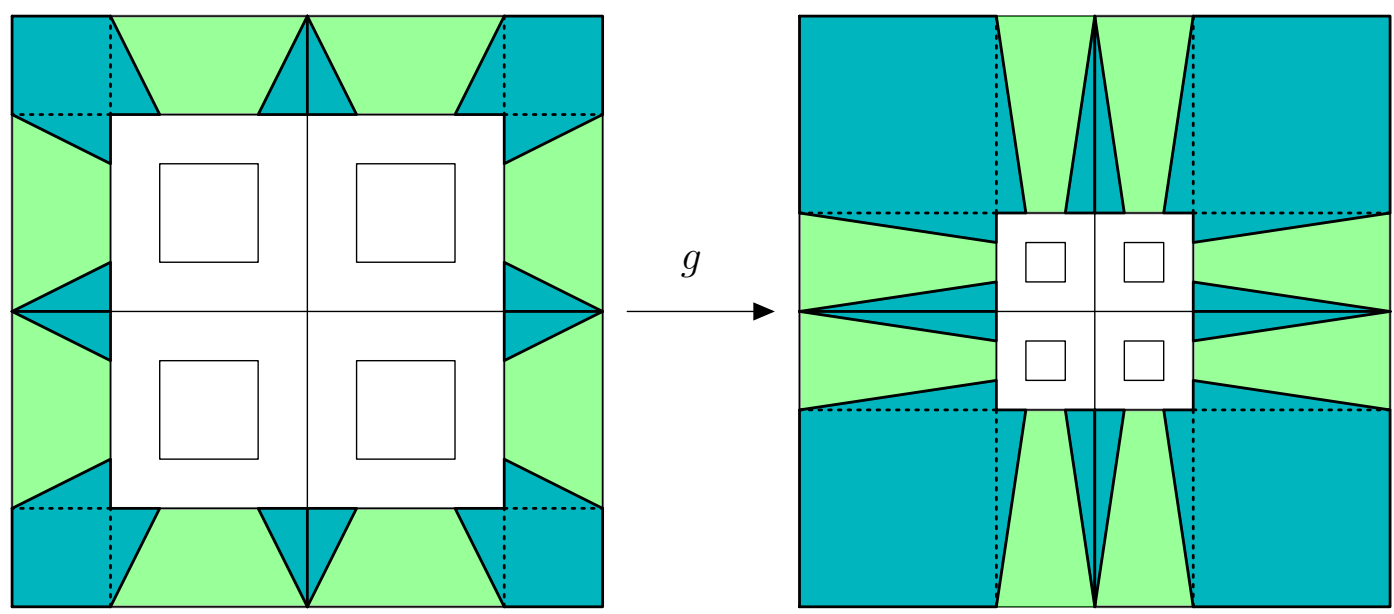

Fig. 5 Homeomorphism $g$ which maps the corners and the sides of $Q_{\boldsymbol{v}(k)}^{\prime} \backslash Q_{\boldsymbol{v}(k)}$ onto corresponing corners and sides of $\tilde{Q}_{\boldsymbol{v}(k)}^{\prime} \backslash \tilde{Q}_{\boldsymbol{v}(k)}$ when $n=2$

The mapping which we have in mind is similar to the one in Fig. 5. However, before giving the explicit construction of this mapping we need to divide each frame $Q_{\boldsymbol{v}(k)}^{\prime} \backslash Q_{\boldsymbol{v}(k)}$ into suitable regions. In each of these regions we then define the mapping separately. For this purpose, we first introduce the definitions of the admissible lattice points and adjacent (admissible) lattice points: 
Admissible lattice points: We define the set of admissible lattice points of the frame $Q_{\boldsymbol{v}(k)}^{\prime} \backslash Q_{\boldsymbol{v}(k)}$ as follows. First we define the set of outer lattice points of $Q_{\boldsymbol{v}(k)}^{\prime} \backslash$ $Q_{\boldsymbol{v}(k)}$ as $P_{O}=P_{O}^{1} \cup P_{O}^{2}$, where

$$
\begin{aligned}
& P_{O}^{1}:=\left\{x \in \partial Q_{\boldsymbol{v}(k)}^{\prime}:\left|x_{1}-\left(z_{\boldsymbol{v}(k)}\right)_{1}\right|=\frac{r_{k-1}}{2} \text { and }\left|x_{2}-\left(z_{\boldsymbol{v}(k)}\right)_{2}\right| \in\left\{\frac{r_{k-1}}{2}, r_{k}, 0\right\}\right\}, \\
& P_{O}^{2}:=\left\{x \in \partial Q_{\boldsymbol{v}(k)}^{\prime}:\left|x_{2}-\left(z_{\boldsymbol{v}(k)}\right)_{2}\right|=\frac{r_{k-1}}{2} \text { and }\left|x_{1}-\left(z_{\boldsymbol{v}(k)}\right)_{1}\right| \in\left\{\frac{r_{k-1}}{2}, r_{k}, 0\right\}\right\} .
\end{aligned}
$$

Similarly, we define the set of inner lattice points of $Q_{\boldsymbol{v}(k)}^{\prime} \backslash Q_{\boldsymbol{v}(k)}$ as $P_{I}=P_{I}^{1} \cup P_{I}^{2}$, where

$$
\begin{aligned}
& P_{I}^{1}:=\left\{x \in \partial Q_{\boldsymbol{v}(k)}:\left|x_{1}-\left(z_{\boldsymbol{v}(k)}\right)_{1}\right|=r_{k} \text { and }\left|x_{2}-\left(z_{\boldsymbol{v}(k)}\right)_{2}\right| \in\left\{r_{k}, \frac{r_{k}}{2} \pm r_{k+1}, 0\right\}\right\}, \\
& P_{I}^{2}:=\left\{x \in \partial Q_{\boldsymbol{v}(k)}:\left|x_{2}-\left(z_{\boldsymbol{v}(k)}\right)_{2}\right|=r_{k} \text { and }\left|x_{1}-\left(z_{\boldsymbol{v}(k)}\right)_{1}\right| \in\left\{r_{k}, \frac{r_{k}}{2} \pm r_{k+1}, 0\right\}\right\} .
\end{aligned}
$$

We define the set of admissible lattice points as the union

$$
P=P_{O} \cup P_{I},
$$

see the left picture in Fig 6.

Adjacent lattice points: We say that two admissible lattice points $p_{1} \in P$ and $p_{2} \in P$ are adjacent if they satisfy the following three conditions

$(\tilde{A} 1)$ the line segment $I:=\left[p_{1}, p_{2}\right]$ is parallel to one of the coordinate axes,

$(\tilde{A} 2) I \subset \overline{Q_{\boldsymbol{v}(k)}^{\prime} \backslash Q_{\boldsymbol{v}(k)}}$, and

$(\tilde{A} 3)$ there is no third lattice point $p \in L$ such that $p \in\left[p_{1}, p_{2}\right]$

or the condition

(A4) up to a relabelling of the points we have $p_{1} \in P_{O}, p_{2} \in P_{I}$, and in addition

$$
\min _{i}\left|\left(p_{1}-p_{2}\right)_{i}\right|=\frac{r_{k}}{2}-r_{k+1} \quad \text { and } \quad \max _{i}\left|\left(p_{1}-p_{2}\right)_{i}\right|=\frac{r_{k-1}}{2}-r_{k} \text {. }
$$

In other words, points $p_{1}$ and $p_{2}$ are adjacent if they are connected by a line segment in $Q_{\boldsymbol{v}(k)}^{\prime} \backslash Q_{\boldsymbol{v}(k)}$ in the picture on the right-hand side in Fig. 6.

Dividing the frame into regions: Next, we define the following collection of line segments

$$
L:=\left\{\left[p_{1}, p_{2}\right]: p_{1} \in P_{O} \text { and } p_{2} \in P_{I} \text { are adjacent lattice points }\right\}
$$

The pair $(P, L)$ can be considered as a planar graph which has 28 components (4 squares, 16 right triangles and 8 isosceles trapezoids) inside $\overline{Q_{\boldsymbol{v}(k)}^{\prime} \backslash Q_{\boldsymbol{v}(k)}}$, see the picture on the righ-hand side in Fig. 6 . 

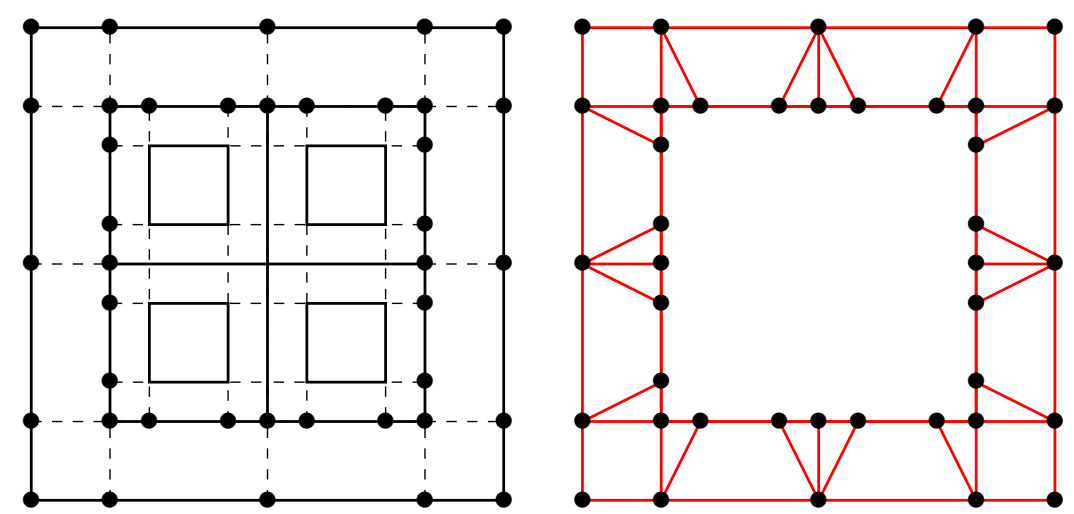

Fig. 6 The set $P$ of admissible lattice points (the left picture) and the graph $(P, L)$ (the right picture)

We call the four squares the vertices of the frame, the 16 triangles the subvertices of the frame and the 8 isosceles trapezoids the sides of the frame. In addition, the union of all the vertices and subvertices of $Q_{\boldsymbol{v}(k)}^{\prime} \backslash Q_{\boldsymbol{v}(k)}$ are called the (two dimensional) corners of $Q_{\boldsymbol{v}(k)}^{\prime} \backslash Q_{\boldsymbol{v}(k)}$. We may define similar kind of graph $(\tilde{P}, \tilde{L})$ also for the frame $\tilde{Q}_{\boldsymbol{v}(k)}^{\prime} \backslash \tilde{Q}_{\boldsymbol{v}(k)}$ and define the vertices, the subvertices, the sides, and the corners of this frame as well.

Definition of $g$ at the vertices: Suppose that

$$
V=Q\left(c, \frac{1}{2}\left(\frac{r_{k-1}}{2}-r_{k}\right)\right)
$$

is one of the vertices of the frame $Q_{\boldsymbol{v}(k)}^{\prime} \backslash Q_{\boldsymbol{v}(k)}$ and suppose that

$$
\tilde{V}=Q\left(\tilde{c}, \frac{1}{2}\left(\frac{\tilde{r}_{k-1}}{2}-\tilde{r}_{k}\right)\right)
$$

is the corresponding vertex of the frame $\tilde{Q}_{\boldsymbol{v}(k)}^{\prime} \backslash \tilde{Q}_{\boldsymbol{v}(k)}$. Then we map $V$ onto corresponding $\tilde{V}$ linearly by setting

$$
g(x)=\left(\frac{x-c}{\frac{r_{k-1}}{2}-r_{k}}\right)\left(\frac{\tilde{r}_{k-1}}{2}-\tilde{r}_{k}\right)+\tilde{c}
$$

for every $x \in V$.

$V$

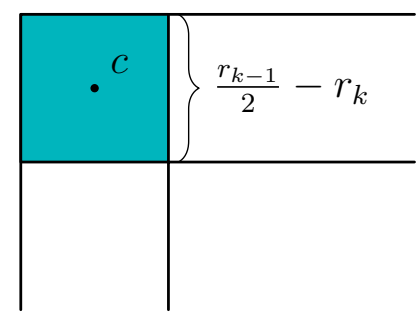

$\tilde{V}$

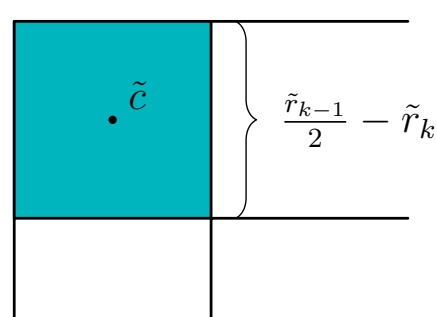

Fig. 7 Homeomorphism $g$ mapping $V$ onto corresponding $\tilde{V}$ 
Then for every $x \in V$ we have the following estimates

$$
\begin{aligned}
& |D g(x)| \sim \frac{\tilde{r}_{k-1}-2 \tilde{r}_{k}}{r_{k-1}-2 r_{k}} \sim \frac{2^{-\beta k}-2^{-\beta(k+1)}}{2^{-k}-2^{-(k+1)}} \sim 2^{k-\beta k}, \quad J_{g}(x) \sim 2^{2(k-\beta k)} \quad \text { and } \\
& K_{O}(x, g) \sim 1 .
\end{aligned}
$$

Definition of $g$ at the subvertices: Let us next consider one of the subvertices $V^{*}$ of the frame $Q_{\boldsymbol{v}(k)}^{\prime} \backslash Q_{\boldsymbol{v}(k)}$ and the corresponding subvertex $\tilde{V}^{*}$ of the frame $\tilde{Q}_{\boldsymbol{v}(k)}^{\prime} \backslash \tilde{Q}_{\boldsymbol{v}(k)}$. We may consider the subvertex $V^{*}$ as a right triangle with a right angle at a point $b=\left(b_{1}, b_{2}\right) \in \mathbb{R}^{2}$ and with both sides parallel to coordinate axis. Respectively, we may consider $\tilde{V}^{*}$ as a right triangle with a right angle at a point $\tilde{b} \in \mathbb{R}^{2}$ and with both sides parallel to coordinate axis. We say that $V^{*}$ is

(1) of type 1 if the lenght of the side of $V^{*}$ which is parallel to $x_{1}$-axis equals $\frac{r_{k-1}}{2}-r_{k}$.

(2) of type 2 if the lenght of the side of $V^{*}$ which is parallel to $x_{2}$-axis equals $\frac{r_{k-1}}{2}-r_{k}$.

$V^{*}$ is of type 1
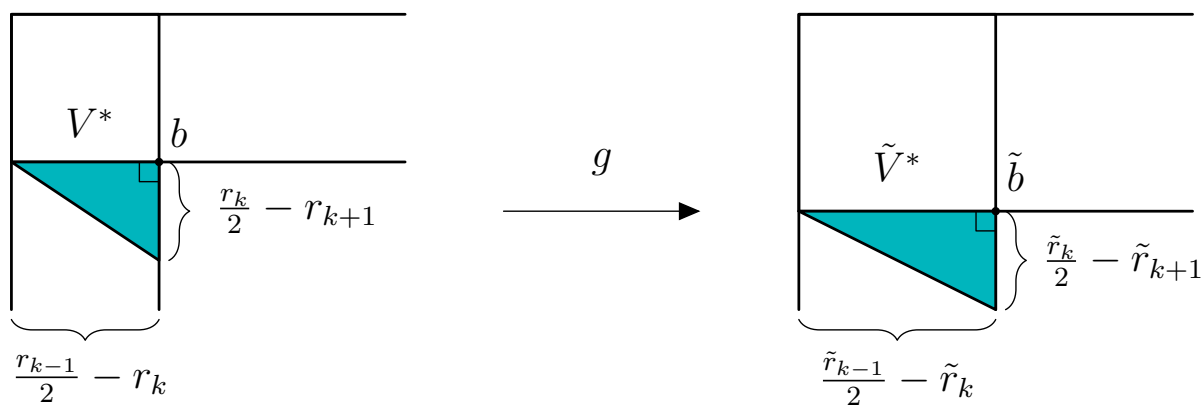

$V^{*}$ is of type 2
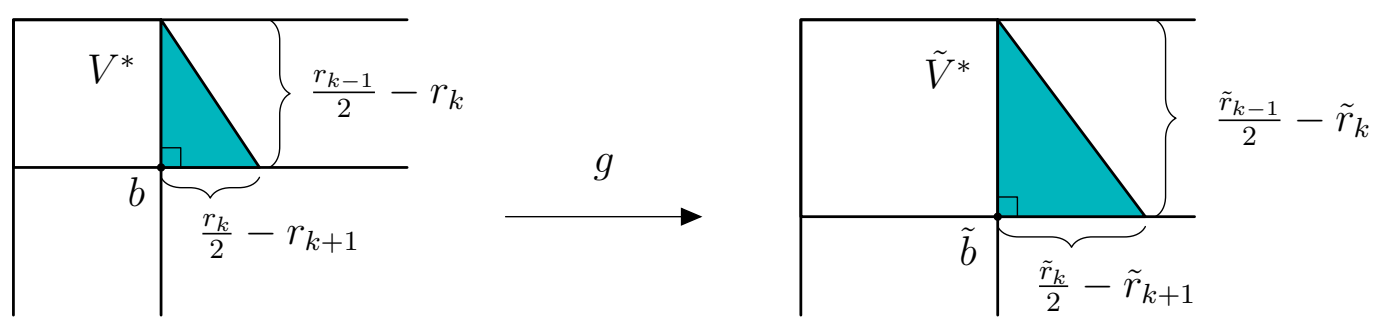

Fig. 8 Homeomorphism $g$ mapping $V^{*}$ onto corresponding $\tilde{V}^{*}$

For every $x \in V^{*}$ we define

$$
g(x)=\left\{\begin{array}{l}
\left(\left(\frac{x_{1}-b_{1}}{\frac{r_{k-1}}{2}-r_{k}}\right)\left(\frac{\tilde{r}_{k-1}}{2}-\tilde{r}_{k}\right),\left(\frac{x_{2}-b_{2}}{\frac{r_{k}}{2}-r_{k+1}}\right)\left(\frac{\tilde{r}_{k}}{2}-\tilde{r}_{k+1}\right)\right)+\tilde{b}, \text { if } V^{*} \text { is of type } 1 \\
\left(\frac{x_{1}-b_{1}}{\frac{r_{k}}{2}-r_{k+1}}\right)\left(\frac{\tilde{r}_{k}}{2}-\tilde{r}_{k+1}\right),\left(\frac{x_{2}-b_{2}}{\frac{r_{k-1}-r_{k}}{2}-r_{k}}\left(\frac{\tilde{r}_{k-1}}{2}-\tilde{r}_{k}\right)\right)+\tilde{b}, \text { if } V^{*} \text { is of type } 2 .
\end{array}\right.
$$


Then for every $x \in V^{*}$ we have the following estimates

$$
\begin{aligned}
& |D g(x)| \sim \max \left\{\frac{\tilde{r}_{k}-2 \tilde{r}_{k+1}}{r_{k}-2 r_{k+1}}, \frac{\tilde{r}_{k-1}-2 \tilde{r}_{k}}{r_{k-1}-2 r_{k}}\right\} \sim \max \left\{2^{k-\beta k}, 2^{k-\beta k}\right\} \sim 2^{k-\beta k}, \\
& J_{g}(x) \sim 2^{2(k-\beta k)} \quad \text { and } \quad K_{O}(x, g) \sim 1 .
\end{aligned}
$$

Definition of $g$ at the sides: Let us next consider one of the sides $S$ of the frame $Q_{\boldsymbol{v}(k)}^{\prime} \backslash Q_{\boldsymbol{v}(k)}$ and the corresponding side $\tilde{S}$ of the frame $\tilde{Q}_{\boldsymbol{v}(k)}^{\prime} \backslash \tilde{Q}_{\boldsymbol{v}(k)}$. We may consider the side $S$ as a isosceles trapezoid with bottom and top sides of lenght $l_{1}=r_{k}$ and $l_{2}=2 r_{k+1}$, respectively. Let us denote the midpoint of the side of length $l_{1}$ by $d=\left(d_{1}, d_{2}\right) \in \partial Q_{\boldsymbol{v}(k)}^{\prime}$. Respectively, we may consider the side $\tilde{S}$ as a isosceles trapezoid with bottom and top sides of lenght $\tilde{l}_{1}=\tilde{r}_{k}$ and $\tilde{l}_{2}=2 \tilde{r}_{k+1}$. Let us denote the midpoint of the side of length $\tilde{l}_{1}$ by $\tilde{d} \in \partial \tilde{Q}_{\boldsymbol{v}(k)}^{\prime}$. We say that $S$ is

(1) of type 1 if the parallel sides of $S$ are parallel to $x_{1}$-axis.

(2) of type 2 if the parallel sides of $S$ are parallel to $x_{2}$-axis.

Then we map $S$ onto corresponding $\tilde{S}$ as follows: we set

$$
s_{1}(t)=\frac{t-d_{1}}{\frac{r_{k-1}}{2}-r_{k}} \quad \text { and } \quad s_{2}(t)=\frac{t-d_{2}}{\frac{r_{k-1}}{2}-r_{k}},
$$

and for every $x \in S$ we define

$$
g(x)=\left(\left(1-s_{2}\left(x_{2}\right)\right)\left(\frac{x_{1}-d_{1}}{r_{k}}\right) \tilde{r}_{k}+s_{2}\left(x_{2}\right)\left(\frac{x_{1}-d_{1}}{r_{k+1}}\right) \tilde{r}_{k+1}, s_{2}\left(x_{2}\right)\left(\frac{\tilde{r}_{k-1}}{2}-\tilde{r}_{k}\right)\right)+\tilde{d}
$$

if $S$ is of type 1, and similarly if $S$ is of type 2 we define

$$
g(x)=\left(s_{1}\left(x_{1}\right)\left(\frac{\tilde{r}_{k-1}}{2}-\tilde{r}_{k}\right),\left(1-s_{1}\left(x_{1}\right)\right)\left(\frac{x_{2}-d_{2}}{r_{k}}\right) \tilde{r}_{k}+s_{1}\left(x_{1}\right)\left(\frac{x_{2}-d_{2}}{r_{k+1}}\right) \tilde{r}_{k+1}\right)+\tilde{d} .
$$

$S$ is of type 1
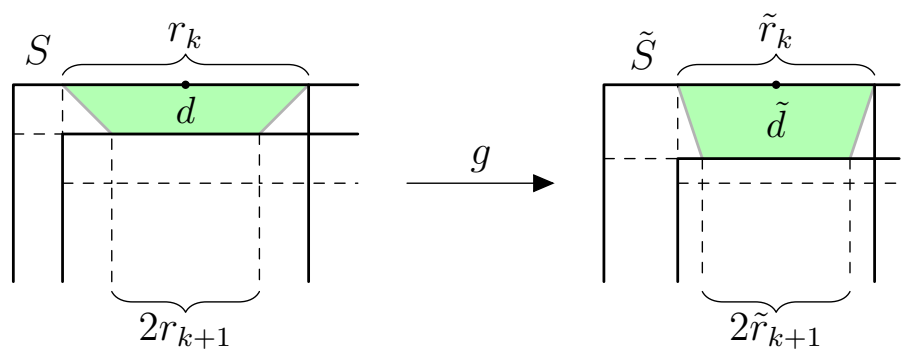

$S$ is of type 2

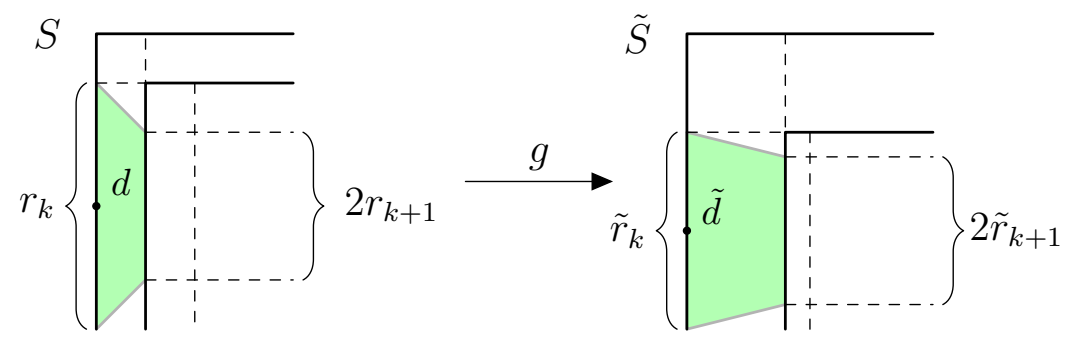


Fig. 9 Homeomorphism $g$ mapping $S$ onto corresponding $\tilde{S}$

Then we have the following estimates

$$
|D g(x)| \sim \max \left\{\frac{\tilde{r}_{k}}{r_{k}}, \frac{\tilde{r}_{k-1}-2 \tilde{r}_{k}}{r_{k-1}-2 r_{k}}\right\} \sim\left\{2^{-\beta k}, 2^{k-\beta k}\right\}=2^{k-\beta k}, \quad J_{g}(x) \sim 2^{-\beta k} 2^{k-\beta k}
$$

and $K_{O}(x, g) \sim 2^{k}$.

5.4. Three dimensional case. Next we give the construction of the homeomorphism $g$ in the case $n=3$. Again, we start the construction by dividing the frame $Q_{\boldsymbol{v}(k)}^{\prime} \backslash Q_{\boldsymbol{v}(k)}$ into different regions:

Admissible lattice points (in three dimensions): We define the set $P_{O}$ of outer lattice points of $Q_{\boldsymbol{v}(k)}^{\prime} \backslash Q_{\boldsymbol{v}(k)}$ as the union

$$
P_{O}=P_{O}^{1,2} \cup P_{O}^{1,3} \cup P_{O}^{2,3},
$$

where

$$
\begin{array}{r}
P_{O}^{i, j}=\left\{x \in \partial Q_{\boldsymbol{v}(k)}^{\prime}:\left|\left(x-z_{\boldsymbol{v}(k)}\right)_{i}\right|,\left|\left(x-z_{\boldsymbol{v}(k)}\right)_{j}\right| \in\left\{0, r_{k}, \frac{r_{k-1}}{2}\right\}\right. \text { and } \\
\left.\left|\left(x-z_{\boldsymbol{v}(k)}\right)_{l}\right|=\frac{r_{k-1}}{2} \text { when } l \in\{1,2,3\} \backslash\{i, j\}\right\} .
\end{array}
$$

Similarly, we define the set of inner lattice points as the union

$$
P_{I}=P_{I}^{1,2} \cup P_{I}^{1,3} \cup P_{I}^{2,3},
$$

where

$$
\begin{aligned}
P_{I}^{i, j}=\left\{x \in \partial Q_{\boldsymbol{v}(k)}:\left|\left(x-z_{\boldsymbol{v}(k)}\right)_{i}\right|,\left|\left(x-z_{\boldsymbol{v}(k)}\right)_{j}\right|\right. & \in\left\{0, \frac{r_{k}}{2} \pm r_{k+1}, r_{k}\right\} \text { and } \\
\left|\left(x-z_{\boldsymbol{v}(k)}\right)_{l}\right| & \left.=r_{k} \text { when } l \in\{1,2,3\} \backslash\{i, j\}\right\} .
\end{aligned}
$$

Then we define the set of admissible lattice points as the union

$$
P=P_{O} \cup P_{I} .
$$

Adjacent lattice points (in three dimensions): We say that two admissible lattice points $p_{1} \in P$ and $p_{2} \in P$ are adjacent if they satisfy the following three conditions

$(\tilde{A} 1)$ the line segment $I:=\left[p_{1}, p_{2}\right]$ is parallel to one of the coordinate axes,

$(\tilde{A} 2) I \subset \overline{Q_{\boldsymbol{v}(k)}^{\prime} \backslash Q_{\boldsymbol{v}(k)}}$, and

$(\tilde{A} 3)$ there is no third lattice point $p \in L$ such that $p \in\left[p_{1}, p_{2}\right]$

or the condition

(A4) up to a relabelling of the points we have $p_{1} \in P_{O}$ and $p_{2} \in P_{I}$, and in addition

$$
\min _{i}\left|\left(p_{1}-p_{2}\right)_{i}\right|=\frac{r_{k}}{2}-r_{k+1} \quad \text { and } \quad \max _{i}\left|\left(p_{1}-p_{2}\right)_{i}\right|=\frac{r_{k-1}}{2}-r_{k} \text {. }
$$

Then we may define the following collection of line segments

$$
L:=\left\{\left[p_{1}, p_{2}\right]: p_{1} \in P \text { and } p_{2} \text { are adjacent lattice points }\right\} .
$$


Admissible loops and tiles: Let us consider a sequence $p_{1}, p_{2}, \ldots, p_{m}, p_{m+1}$ of points in $P$ such that

(1) $p_{i}$ and $p_{i+1}$ are adjacent lattice points for all $i=1, \ldots, m$,

(2) $p_{1}=p_{m+1}$, and

(3) $p_{i} \neq p_{j}$ for all distinct indeces $i, j \in\{1,2, \ldots, m\}$.

Then the polyline $\left[p_{1}, p_{2}, \ldots, p_{m}, p_{m+1}\right]$ defines a closed Jordan curve which we call an $m$-loop. In addition, all the 3-loops and 4-loops are called admissible loops. We may notice that for every admissible loop $J$ there is a unique 2-dimensional hyperplane $H$ such that $J \subset H$. In addition, $J$ divides the plane $H$ into exactly two connected components of $H$. One of these components is bounded and another one unbounded in $H$. We call the closure of the bounded component of $H \backslash J$ as a tile. The set of all tiles of the frame $Q_{\boldsymbol{v}(k)}^{\prime} \backslash Q_{\boldsymbol{v}(k)}$ we denote by $\mathcal{F}_{\boldsymbol{v}(k)}$. We may observe that up to a translation and a three dimensional rotation there are 12 different type of tiles. More precisely, every tile in $\mathcal{F}_{\boldsymbol{v}(k)}$ is either

(1) one of the following four squares

$\mathrm{T} 1$

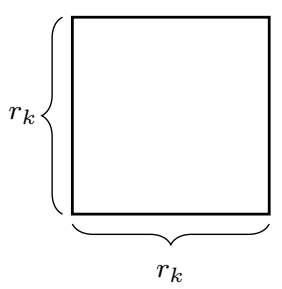

T2

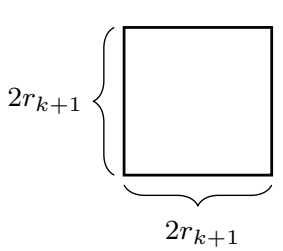

T3

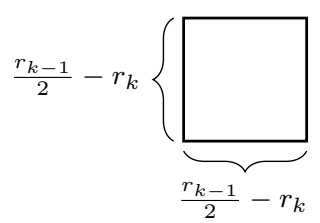

T4

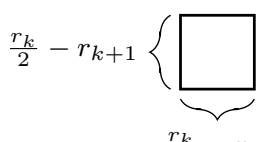

Fig. 10 Tiles T1-T4 are squares

(2) or one of the following two rectangles

T5

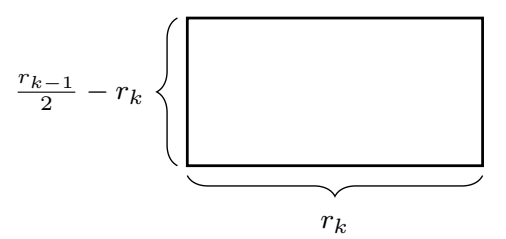

T6

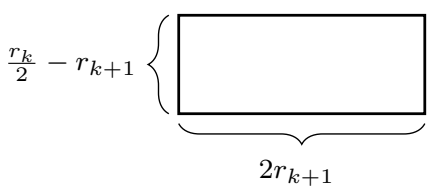

Fig. 11 Tiles T5-T6 are rectangles

(3) or one of the following three triangles 


\section{T8}
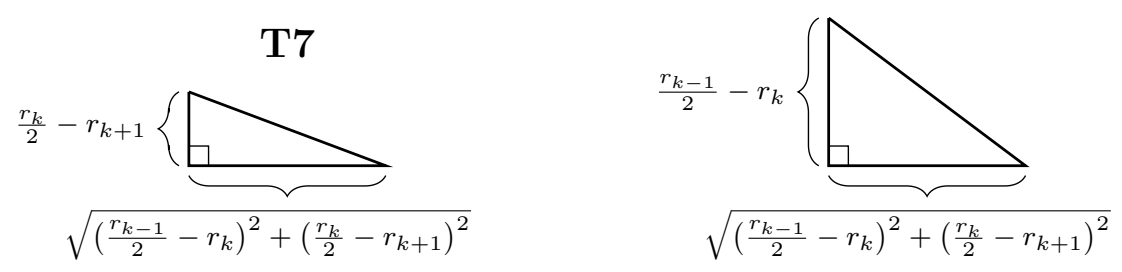

T9

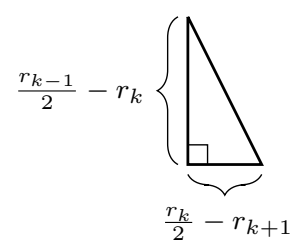

Fig. 12 Tiles T7-T9 are right triangles

(4) or one of the following three isosceles trapezoids
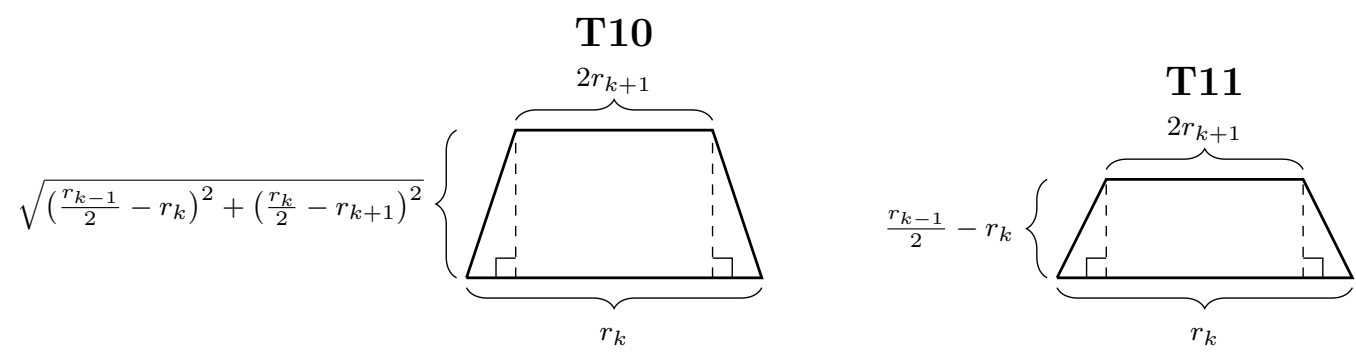

\section{T12}

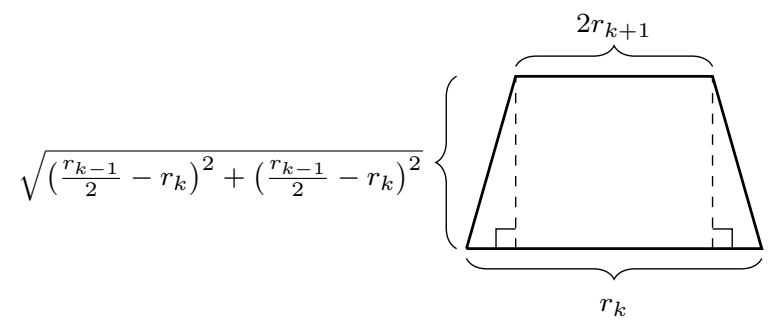

Fig. 13 Tiles T10-T12 are isosceles trapezoids

We define the set $\tilde{\mathcal{F}}_{\boldsymbol{v}(k)}$ of all filled admissible loops of the frame $\tilde{Q}_{\boldsymbol{v}(k)}^{\prime} \backslash \tilde{Q}_{\boldsymbol{v}(k)}$ similarly. It is easy to see that there is a natural one-to-one correspondence between the elements of $\mathcal{F}_{\boldsymbol{v}(k)}$ and $\tilde{\mathcal{F}}_{\boldsymbol{v}(k)}$. 


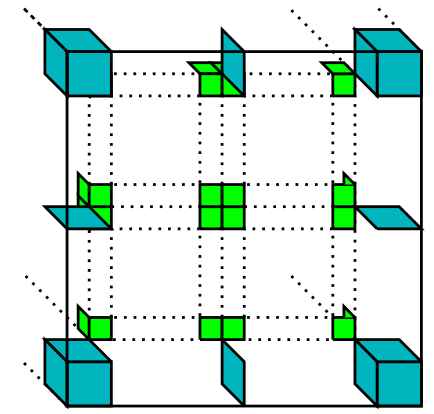

Fig. 14 Some of the T3-tiles (blue slabs) and T4-tiles (green slabs) of the frame $Q_{\boldsymbol{v}(k)}^{\prime} \backslash Q_{\boldsymbol{v}(k)}$. One may count that for each frame the total numbers of T3-tiles and T4-tiles equals 60 and 96

Dividing the frame into regions: One may observe that the set

$$
\left(Q_{\boldsymbol{v}(k)}^{\prime} \backslash Q_{\boldsymbol{v}(k)}\right) \backslash\left(\bigcup_{F \in \mathcal{F}_{\boldsymbol{v}(k)}} F\right)
$$

has exactly 320 connected components. We denote the set of these 320 components by $\mathcal{V}_{\boldsymbol{v}(k)}$. Then we may observe that the set $\mathcal{V}_{\boldsymbol{v}(k)}$ has six different type of elements. More precisely, the elements of $\mathcal{V}_{\boldsymbol{v}(k)}$ consist of 8 vertices, 48 subvertices, 96 sub-subvertices, 48 edges, 96 subedges, and 24 faces which can be defined as follows:

(1) We call a set $V \in \mathcal{V}_{\boldsymbol{v}(k)}$ a vertex if its boundary consists of six T3-tiles (see Fig. 15). Each frame has 8 vertices. Four of these vertices (the four blue cubes) can be found in Fig. 14.

(2) We call a set $V^{*} \in \mathcal{V}_{\boldsymbol{v}(k)}$ a subvertex if its boundary consists of one T3-tile, two T8-tiles, and two T9-tiles (see Fig. 15). These subvertices are located as follows:

(2a) For each vertex $V \in \mathcal{V}_{\boldsymbol{v}(k)}$ there are 3 subvertices each of which is lying on one of the 3 faces of $V$ that are not contained in $\partial Q_{\boldsymbol{v}(k)}^{\prime}$. The total number of this type of subvertices equals $3 \times 8=24$.

(2b) In addition, there are 2 subvertices (pointing at opposite directions) located close to the midpoint of each of the 12 edges of the big cube in Fig. 16. Each of these subvertices lies on a square similar to the four isolated blue squares in Fig. 14. The total number of this type of subvertices equals $2 \times 12=24$.

We call two distinct subvertices $V_{1}^{*}$ and $V_{2}^{*}$ neighbors if there is a line segment parallel to coordinate axis which connects one of the triangular faces of $V_{1}^{*}$ to one of the triangular faces of $V_{2}^{*}$ without intersecting any other tile. We point out that for every subvertex there exists exactly one neighboring subvertex.

(3) We call a set $V^{* *} \in \mathcal{V}_{\boldsymbol{v}(k)}$ a sub-subvertex if its boundary consists of one T4-tile, two T7-tiles, and two T9-tiles (see Fig. 15). Every frame contains $16 \times 6=96$ sub-subvertices, and each of these sub-subvertices lies on a square similar to the green squares in Fig. 14. We call two distinct sub-subvertices $V_{1}^{* *}$ and $V_{2}^{* *}$ neighbors if there is a line segment parallel to coordinate axis which connects one of the triangular faces of $V_{1}^{* *}$ to one of the triangular faces of $V_{2}^{* *}$ without 
intersecting any other tile. We point out that every sub-subvertex has exactly two neighbors.

(4) We call a set $E \in \mathcal{V}_{\boldsymbol{v}(k)}$ an edge if its boundary consists of one T5-tile, two T8-tiles, one T11-tile, and one T12-tile (see Fig. 15). There are two edges connecting every two neighboring subvertices, and there are no other type of edges. Therefore, the total number of edges equals 48 , which can be easily seen from Fig. 14.

(5) We call a set $E^{*} \in \mathcal{V}_{\boldsymbol{v}(k)}$ a subedge if its boundary consists of one T6-tile, two T7-tiles, one T10-tile, and one T11-tile (see Fig 15). There is one subedge connecting every two neighboring sub-subvertices, and there are no other type of subedges. Therefore, the total number of subedges equals $6 \times 16=96$, which can be easily seen from Fig. 14 .

(6) We call a set $F \in \mathcal{V}_{\boldsymbol{v}(k)}$ a face if its boundary consists of one T1-tile, one T2-tile, and four T10-tiles (see Fig. 15). These sets correspond the 4 empty pieces on each of the 6 sides of the cube in Fig. 16.

Up to a rotation and a translation each of these elements is equivalent to one of the sets in Fig. 15. Respectively, we may define the set $\tilde{\mathcal{V}}_{\boldsymbol{v}(k)}$ as a set of the components of

$$
\left(\tilde{Q}_{\boldsymbol{v}(k)}^{\prime} \backslash \tilde{Q}_{\boldsymbol{v}(k)}\right) \backslash\left(\bigcup_{\tilde{F} \in \tilde{\mathcal{F}}_{\boldsymbol{v}(k)}} \tilde{F}\right) .
$$

There is also a natural one-to-one correspondence between the elements of $\mathcal{V}_{\boldsymbol{v}(k)}$ and $\tilde{\mathcal{V}}_{\boldsymbol{v}(k)}$.

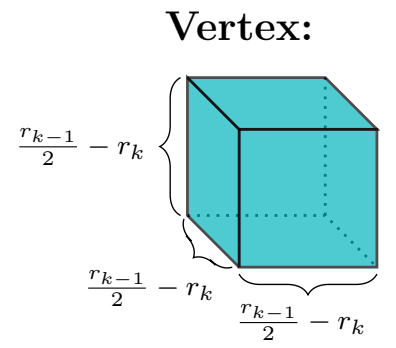

Edge:

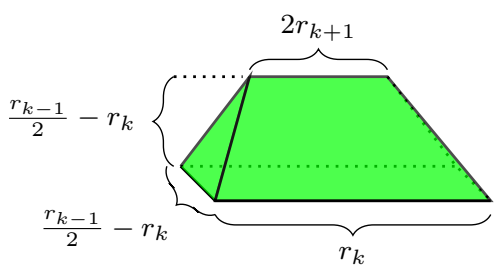

Subvertex:

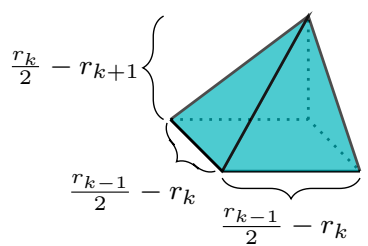

Sub-subvertex:

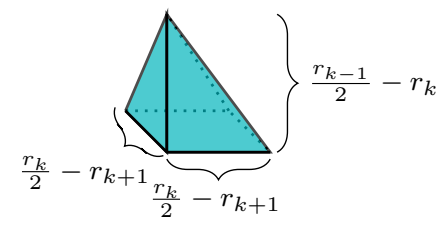

Subedge:

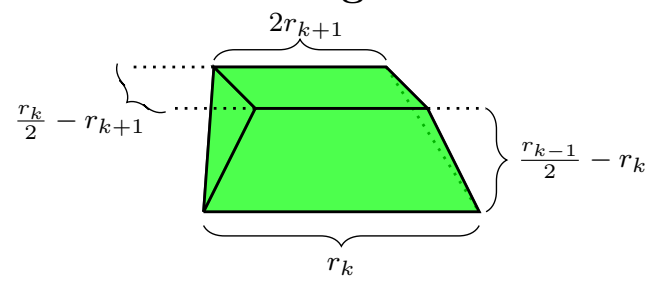

Face:

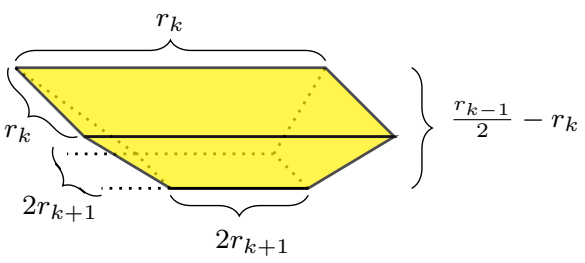

Fig. 15 A Vertex, a subvertex, a sub-subvertex, an edge, a subedge and a face of the frame $Q_{\boldsymbol{v}(k)}^{\prime} \backslash Q_{\boldsymbol{v}(k)}$ 
From now on we denote the union of all the vertices, the subvertices and the subsubvertices of $Q_{\boldsymbol{v}(k)}^{\prime} \backslash Q_{\boldsymbol{v}(k)}$ by $V_{\boldsymbol{v}(k)}$, the union of all the edges and the subedges of $Q_{\boldsymbol{v}(k)}^{\prime} \backslash Q_{\boldsymbol{v}(k)}$ by $E_{\boldsymbol{v}(k)}$, and the union of all the faces of $Q_{\boldsymbol{v}(k)}^{\prime} \backslash Q_{\boldsymbol{v}(k)}$ by $F_{\boldsymbol{v}(k)}$. The corresponding sets $\tilde{V}_{\boldsymbol{v}(k)}, \tilde{E}_{\boldsymbol{v}(k)}$ and $\tilde{F}_{\boldsymbol{v}(k)}$ of the frame $\tilde{Q}_{\boldsymbol{v}(k)}^{\prime} \backslash \tilde{Q}_{\boldsymbol{v}(k)}$ are defined respectively.

Defining the homeomorphism $g$ in three dimensions: The definition of the homeomorphism $g:[-1,1]^{3} \rightarrow[-1,1]^{3}$ is analogical to the two dimensional case and therefore we leave the explicit formulation of the mapping for the reader. The main idea of the construction is to map each element of $\mathcal{V}_{\boldsymbol{v}(k)}$ onto corresponding element of $\tilde{\mathcal{V}}_{\boldsymbol{v}(k)}$ in a natural way. More precisely, on each vertex, subvertex and sub-subvertex we define $g$ as a suitable affine mapping. In addition, on each edge, subedge and face the coordinate functions of $g$ can be defined as variable convex combinations of some affine functions. More precisely, up to some translations and rotations of the space on each edge, subedge and face we may write $g$ as

$$
g(x)=\left(g_{1}\left(x_{1}, x_{3}\right), g_{2}\left(x_{2}, x_{3}\right), g_{3}\left(x_{3}\right)\right),
$$

where $g_{3}$ is an affine function, and for each fixed $x_{3}$ the functions $g_{1}\left(\cdot, x_{3}\right)$ and $g_{2}\left(\cdot, x_{3}\right)$ are affine with respect to the first variable. Moreover, as the formula (5.3) suggests, it is possible to define $g$ so that up to a change of orthogonal coordinate systems $D g(x)$ will define an upper diagonal matrix at each point of these sets. This makes estimating the Jacobian easy at each point of the sets. It is easy to check that we may construct $g$ such a way that it defines a homeomorphism. If we construct $g$ this way it will lead us to the following estimates:

$$
\begin{aligned}
& |D g(x)| \sim\left\{\begin{array}{ll}
2^{k-\beta k}, & \text { if } x \in V_{\boldsymbol{v}(k)} \\
2^{k-\beta k}, & \text { if } x \in E_{\boldsymbol{v}(k)} \\
2^{k-\beta k}, & \text { if } x \in F_{\boldsymbol{v}(k)}
\end{array} \quad, \quad J_{g}(x) \sim\left\{\begin{array}{ll}
2^{3(k-\beta k)}, & \text { if } x \in V_{\boldsymbol{v}(k)} \\
2^{2(k-\beta k)} 2^{-\beta k}, & \text { if } x \in E_{\boldsymbol{v}(k)} \\
2^{k-\beta k} 2^{-2 \beta k}, & \text { if } x \in F_{\boldsymbol{v}(k)}
\end{array} \quad\right. \text { and }\right. \\
& \left|D^{\sharp} g(x)\right| \sim \begin{cases}2^{2(k-\beta k)}, & \text { if } x \in V_{\boldsymbol{v}(k)} \\
2^{2(k-\beta k)}, & \text { if } x \in E_{\boldsymbol{v}(k)} \\
2^{k-\beta k} 2^{-\beta k}, & \text { if } x \in F_{\boldsymbol{v}(k)},\end{cases}
\end{aligned}
$$

and also

$$
K_{O}(x, g) \sim\left\{\begin{array} { l l } 
{ 1 , } & { \text { if } x \in V _ { \boldsymbol { v } ( k ) } } \\
{ 2 ^ { k } , } & { \text { if } x \in E _ { \boldsymbol { v } ( k ) } } \\
{ 2 ^ { 2 k } , } & { \text { if } x \in F _ { \boldsymbol { v } ( k ) } }
\end{array} \quad \text { and } \quad K _ { I } ( x , g ) \sim \left\{\begin{array}{ll}
1, & \text { if } x \in V_{\boldsymbol{v}(k)} \\
2^{2 k}, & \text { if } x \in E_{\boldsymbol{v}(k)} \\
2^{k}, & \text { if } x \in F_{\boldsymbol{v}(k)} .
\end{array}\right.\right.
$$

Similarly it is possible to estimate the derivative of the inverse as follows:

$$
\begin{aligned}
& \left|D g^{-1}(y)\right| \sim\left\{\begin{array} { l l } 
{ 2 ^ { \beta k - k } , } & { \text { if } y \in \tilde { V } _ { \boldsymbol { v } ( k ) } } \\
{ 2 ^ { \beta k } , } & { \text { if } y \in \tilde { E } _ { \boldsymbol { v } ( k ) } } \\
{ 2 ^ { \beta k } , } & { \text { if } y \in \tilde { F } _ { \boldsymbol { v } ( k ) } }
\end{array} \quad J _ { g ^ { - 1 } } ( y ) \sim \left\{\begin{array}{ll}
2^{3(\beta k-k)}, & \text { if } y \in \tilde{V}_{\boldsymbol{v}(k)} \\
2^{2(\beta k-k)} 2^{\beta k}, & \text { if } y \in \tilde{E}_{\boldsymbol{v}(k)} \\
2^{(\beta k-k)} 2^{2 \beta k}, & \text { if } y \in \tilde{F}_{\boldsymbol{v}(k)}
\end{array} \quad\right.\right. \text { and } \\
& \left|D^{\sharp} g^{-1}(y)\right| \sim \begin{cases}2^{2(\beta k-k)}, & \text { if } y \in \tilde{V}_{\boldsymbol{v}(k)} \\
2^{(\beta k-k)} 2^{\beta k}, & \text { if } y \in \tilde{E}_{\boldsymbol{v}(k)} \\
2^{2 \beta k}, & \text { if } y \in \tilde{F}_{\boldsymbol{v}(k)},\end{cases}
\end{aligned}
$$


and then for the distortions we have

$$
K_{O}\left(y, g^{-1}\right) \sim\left\{\begin{array} { l l } 
{ 1 , } & { \text { if } y \in \tilde { V } _ { \boldsymbol { v } ( k ) } } \\
{ 2 ^ { 2 k } , } & { \text { if } y \in \tilde { E } _ { \boldsymbol { v } ( k ) } } \\
{ 2 ^ { k } , } & { \text { if } y \in \tilde { F } _ { \boldsymbol { v } ( k ) } }
\end{array} \quad \text { and } \quad K _ { I } ( y , g ^ { - 1 } ) \sim \left\{\begin{array}{ll}
1, & \text { if } y \in \tilde{V}_{\boldsymbol{v}(k)} \\
2^{k}, & \text { if } y \in \tilde{E}_{\boldsymbol{v}(k)} \\
2^{2 k}, & \text { if } y \in \tilde{F}_{\boldsymbol{v}(k)} .
\end{array}\right.\right.
$$

5.5. Definition of the corners in three dimensions. Recall from section 5.4 the definitions of the sets $V_{\boldsymbol{v}(k)}$ (union of all the vertices, the subvertices and the sub-subvertices of the frame) and $E_{\boldsymbol{v}(k)}$ (union of all the edges and subedges of the frame). We define the (three dimensional) corners of the frame $Q_{\boldsymbol{v}(k)}^{\prime} \backslash Q_{\boldsymbol{v}(k)}$ as the union

$$
C_{\boldsymbol{v}(k)}:=V_{\boldsymbol{v}(k)} \cup E_{\boldsymbol{v}(k)},
$$

see Fig. 16. The corners $\tilde{C}_{\boldsymbol{v}(k)}$ of the frame $\tilde{Q}_{\boldsymbol{v}(k)}^{\prime} \backslash \tilde{Q}_{\boldsymbol{v}(k)}$ can be defined similarly.

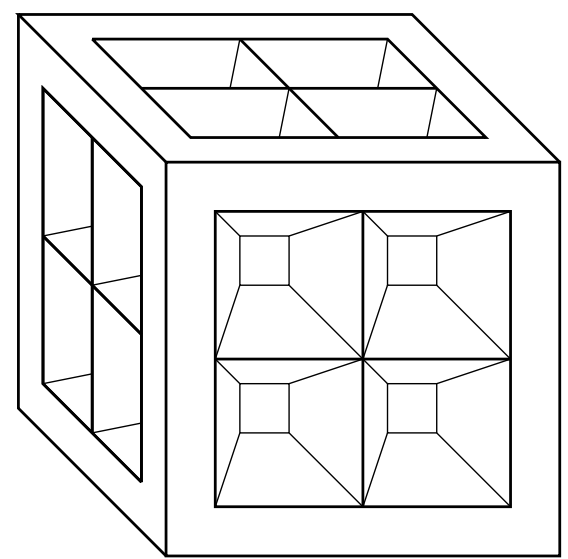

Fig. 16 Three dimensional corners $C_{\boldsymbol{v}(k)}$ of $Q_{\boldsymbol{v}(k)}^{\prime} \backslash Q_{\boldsymbol{v}(k)}$

It is easy to see that we can use (5.1) and (5.2) to estimate the size of the set $C_{\boldsymbol{v}(k)}$ as follows

and hence

$$
\mathcal{L}^{3}\left(C_{\boldsymbol{v}(k)}\right) \leq C r_{k-1}\left(\frac{r_{k-1}}{2}-r_{k}\right)^{2} \leq C 2^{-5 k}
$$

$$
\mathcal{L}^{3}\left(\bigcup_{\boldsymbol{v}(k) \in \mathbb{V} k} C_{\boldsymbol{v}(k)}\right) \leq 2^{3 k} \mathcal{L}^{3}\left(C_{\boldsymbol{v}(k)}\right) \leq C 2^{-2 k}
$$

\section{EXPliCit CONSTRUCTION OF THE BI-LiPsCHITZ MAP IN THREE DIMENSIONS}

Let us now consider the Cantor set $C_{B}:=C\left[\left\{b_{k}\right\}_{k=0}^{\infty}\right]$ of zero measure defined by the sequence

$$
b_{k}=2^{-10 k}
$$

and suppose the Cantor set $C_{A}:=C_{A}\left[\left\{a_{k}\right\}_{k=0}^{\infty}\right]$ is defined as in section 5 .

We express points in coordinates as $(x, y, z)$. 


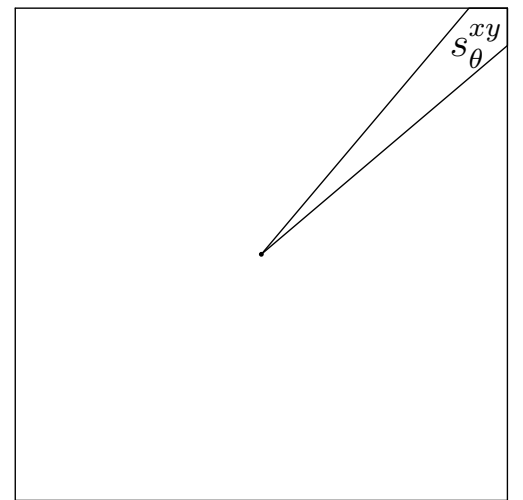

Fig. 17 Definition of sector $\mathcal{S}_{\theta}$.

Let $\theta>0$ be small enough (we give the exact condition later) but fixed. We use $\mathcal{S}_{\theta}$ to denote a specific "sector" of with an opening angle $\theta$ :

$$
\mathcal{S}_{\theta}:=\left\{x \in \mathbb{R}^{n}: 1-\frac{\theta}{2} \leq \arctan (y / x) \leq 1+\frac{\theta}{2}\right\},
$$

i.e., $\mathcal{S}_{\theta}=\left\{(x, y, z) \in[-1,1]^{3}:(x, y) \in s_{\theta}^{x y}\right\}$ where the set $s_{\theta}^{x y}$ can be seen in Fig. 17 .

Suppose $L:[-1,1]^{3} \rightarrow[-1,1]^{3}$ is a given bi-Lipschitz map which takes the Cantor set $C_{B}$ onto corresponding Cantor's tower $C_{B}^{T}$, and let $g$ be the homeomorphism defined in section 5 . Then we define the set of bad points (related to mapping $L$ ) as

$$
\mathbb{B A D}:=\left\{x \in(-1,1)^{3}: L(g(x)) \in \mathcal{S}_{\theta}\right\},
$$

and for each $k \in\{1,2, \ldots\}$ we define the set of bad points in $k$-th step as

$$
\begin{aligned}
\mathbb{B A D}_{k} & :=\left\{x \in \bigcup_{\boldsymbol{v}(k) \in \mathbb{V}^{k}} Q_{\boldsymbol{v}(k)}^{\prime} \backslash Q_{\boldsymbol{v}(k)}: L(g(x)) \in \mathcal{S}_{\theta}\right\} \\
& =\left\{x \in \bigcup_{\boldsymbol{v}(k) \in \mathbb{V}^{k}} Q_{\boldsymbol{v}(k)}^{\prime} \backslash Q_{\boldsymbol{v}(k)}: x \in \mathbb{B} \mathbb{A D}\right\} .
\end{aligned}
$$

Our goal is to find a bi-Lipschitz map $L:[-1,1]^{3} \rightarrow[-1,1]^{3}$ such that the following conditions hold:

(1) Mapping $L$ takes $C_{B}$ onto $C_{B}^{T}$.

(2) We have

$$
(L \circ g)^{-1}\left(\mathcal{S}_{\theta} \cap\left(\tilde{Q}_{\boldsymbol{v}(k)}^{\prime} \backslash \bigcup_{\boldsymbol{v}(k+1) \in \mathbb{V}^{k+1}} \tilde{Q}_{\boldsymbol{v}(k+1)}^{\prime}\right)\right) \subset \bigcup_{\boldsymbol{v}(k) \in \mathbb{V}^{k}} C_{\boldsymbol{v}(k)} .
$$

In other words, the preimage $(L \circ g)^{-1}\left(\mathcal{S}_{\theta}\right)$ is contained in the union of the corners. This can be written equivalently as follows:

$$
\mathbb{B A D}_{k} \subset C_{\boldsymbol{v}(k)} \text {. }
$$

(3) Mapping $L$ maps $\tilde{Q}_{\boldsymbol{v}(k)}$ onto the cube $L\left(\tilde{Q}_{\boldsymbol{v}(k)}\right)$ of the same size.

We know that the corners of $C_{A}$ are mapped onto the corners of $C_{B}$ by $g$. Hence our mapping $L$ has to map the corners of $C_{B}$ onto a set which contains $\mathcal{S}_{\theta}$. Recall that corners are defined in (5.8) (see also Fig. 16) and hence we require that the images 
of the red pipes (in the Fig. 18 below) under mapping $L$ do not intersect $\mathcal{S}_{\theta}$. We will also prescribe the values of $L$ on the boundaries of $\tilde{Q}_{\boldsymbol{v}}$ so it is easy to glue mappings together.

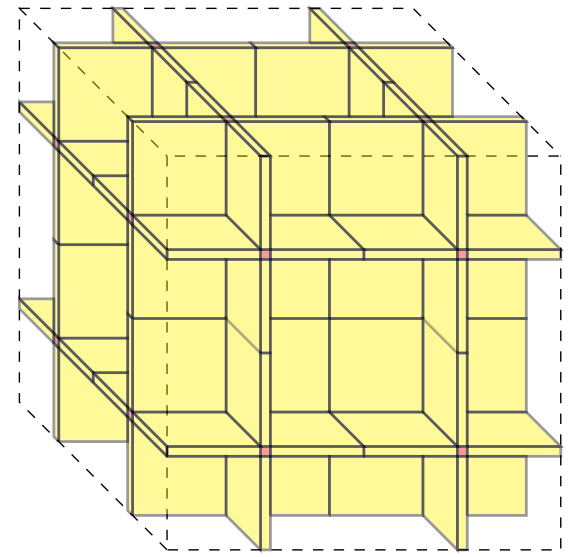

Fig. 18 The sets $\tilde{E}_{\boldsymbol{v}(k)}$ (yellow slabs), the sets $\tilde{F}_{\boldsymbol{v}(k)}$ (red pipes) and the sets $\tilde{V}_{\boldsymbol{v}(k)}$ (empty space between the slabs) when we are close to the center of the cube $\tilde{Q}_{\boldsymbol{v}(k)}$

To define the bi-Lipschitz map $L:[-1,1]^{3} \rightarrow[-1,1]^{3}$ we first define a bi-Lipschitz map $L_{1}:[-1,1]^{3} \rightarrow[-1,1]^{3}$ satisfying the conditions (1)-(3) above for $k=1$. After this we iterate this procedure in the following steps and check that the bi-Lipschitz constant stays the same in all steps of the iteration. Moreover, we also need to check that the boundary values of all the iterations $L_{k}$ will coincide. We briefly outline this below:

Outline of the construction of the mapping $L$. Our mapping will be a composition of four bi-Lipschitz mappings

$$
L_{1}:=L_{x} \circ L_{y} \circ L_{z} \circ L_{p} .
$$

The mapping $L_{p}$ prepares the correct boundary values and it is identity in most places

$$
L_{p}(x, y, z)=(x, y, z) \text { for every }(x, y, z) \in[-1,1]^{2} \times\left[-1+\frac{1}{20}, 1-\frac{1}{20}\right],
$$

especially it is identity on the cubes $\tilde{Q}_{\boldsymbol{v}(1)}$ and in their neighborhood. Furthermore, it moves the small two dimensional red squares on top and bottom of Fig. 18 (corresponding to $z= \pm 1$ ) to other places so that their image does not intersect $\mathcal{S}_{\theta} \cap \partial[-1,1]^{3}$ (more details are given below). Then, we define a mapping $L_{z}$ which moves the 8 cubes $\tilde{Q}_{\boldsymbol{v}(1)}$ in the $z$-direction so that their $z$-coordinate becomes

$$
\left\{-\frac{7}{8},-\frac{5}{8},-\frac{3}{8},-\frac{1}{8}, \frac{1}{8}, \frac{3}{8}, \frac{5}{8}, \frac{7}{8}\right\}
$$

so they correspond to the $z$-coordinates of the tower formation (see Fig. 3 and Fig. 4) in dimension $n=3$. The image under $L_{z}$ of $\tilde{Q}_{\boldsymbol{v}(1)}$ and the red pipes from Fig. 18 is shown in Fig. 19 below (we show the projection to $x z$-plane). It is easy to see that the projections of $L_{z}\left(\tilde{Q}_{\boldsymbol{v}(1)}\right)$ to $z$-coordinate are disjoint and far away (their size is in fact much smaller than on this sketch). 

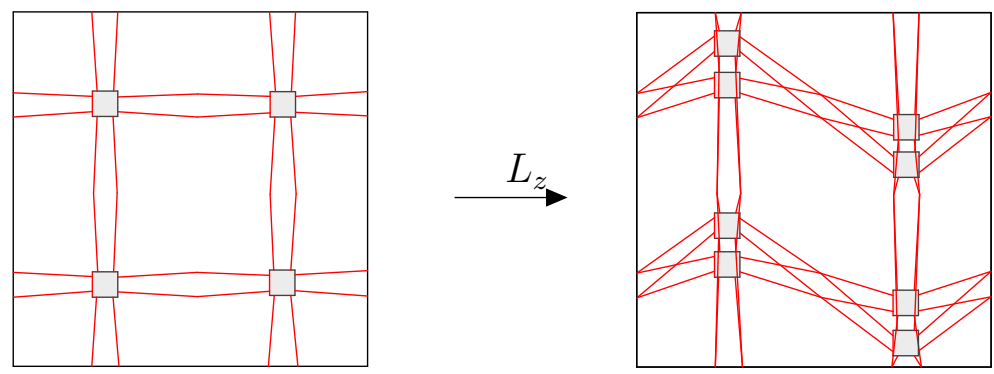

Fig. $19 L_{z}$ maps $z$ coordinates of $\tilde{Q}_{\boldsymbol{v}(1)}$ to tower formation (projection to $x z$-plane)

Then the mapping $L_{y}$ moves the cubes $L_{z}\left(\tilde{Q}_{\boldsymbol{v}(1)}\right)$ in the $y$-direction to the center so that their $y$-coordinate becomes roughly 0 . Then the mapping $L_{x}$ moves the cubes $L_{y}\left(L_{z}\left(\tilde{Q}_{\boldsymbol{v}(1)}\right)\right)$ in the $x$-direction to the center. In this way we map $\tilde{Q}_{\boldsymbol{v}(1)}$ into the tower formation.

Explicit construction of the mapping $L$. Let us now show in detail that we can do the construction, described above, by using bi-Lipschitz maps and that (6.1) will be satisfied at the end if we do this construction in a suitable way.

Let

$$
t \in\left[-\frac{2}{3}+\frac{1}{100}, \frac{2}{3}-\frac{1}{100}\right] \text { and } s \in\left[-\frac{7}{16}+\frac{1}{100}, \frac{7}{16}-\frac{1}{100}\right] .
$$

We define piecewise linear functions $h_{t}, g_{t}:[-1,1] \rightarrow[-1,1]$ such that $h_{t}(-1)=$ $g_{t}(-1)=-1, h_{t}(1)=g_{t}(1)=1$,

$$
\begin{aligned}
& h_{s}(x)=x+s \text { for } s>0 \text { and } x \in\left[-\frac{1}{2}-\frac{1}{16},-\frac{1}{2}+\frac{1}{16}\right], \\
& h_{s}(x)=x+s \text { for } s<0 \text { and } x \in\left[\frac{1}{2}-\frac{1}{16}, \frac{1}{2}+\frac{1}{16}\right], \\
& g_{t}(x)=x+t \text { for } t>0 \text { and } x \in\left[-1+\frac{1}{10}, 1-\frac{2}{3}\right], \\
& g_{t}(x)=x+t \text { for } t<0 \text { and } x \in\left[-1+\frac{2}{3}, 1-\frac{1}{10}\right],
\end{aligned}
$$

and $h_{s}, g_{t}$ are linear on the remaining intervals (see Fig. 20).
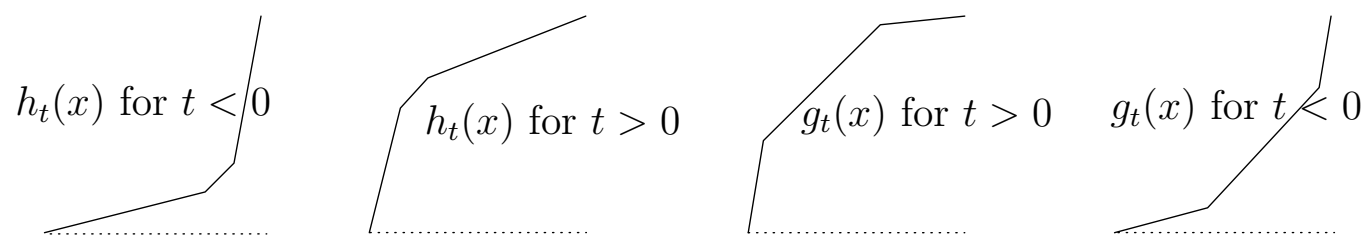

Fig. 20 Graphs of $h_{t}(x)$ and $g_{t}(x)$

It is easy to see that $h_{s}(x)$ and $g_{t}(x)$ are bi-Lipschitz functions. We would like to shift point $x=-\frac{1}{2}$ close to 0 with $g_{t}, t>0$, and point $x=\frac{1}{2}$ close to 0 with $g_{t}$, $t<0$. Moreover, by $h_{s}, s>0$, we would like to shift point $x=-\frac{1}{2}$ to points with coordinates $\left\{-\frac{3}{8},-\frac{1}{8}, \frac{5}{8}, \frac{7}{8}\right\}$ and by $h_{s}, s<0$, point $x=\frac{1}{2}$ to points with coordinates $\left\{-\frac{7}{8},-\frac{5}{8}, \frac{1}{8}, \frac{3}{8}\right\}$ (see (6.3) and Fig. 19).

Let

$$
s_{z}, s_{y}, s_{x}:[-1,1]^{2} \rightarrow\left[-\frac{2}{3}+\frac{1}{100}, \frac{2}{3}-\frac{1}{100}\right]
$$


be fixed Lipschitz mappings such that

$$
s_{a}(x, y)=0 \text { for every }(x, y) \in \partial[-1,1]^{2} \text { and } a \in\{x, y, z\} .
$$

Let us define

$$
\begin{aligned}
& L_{z}(x, y, z)=\left(x, y, h_{s_{z}(x, y)}(z)\right), \\
& L_{y}(x, y, z)=\left(x, g_{s_{y}(x, z)}(y), z\right) \text { and } \\
& L_{x}(x, y, z)=\left(g_{s_{x}(y, z)}(x), y, z\right) .
\end{aligned}
$$

It is not difficult to see that these mappings are Lipschitz, they map $[-1,1]^{3}$ onto $[-1,1]^{3}$ and using (6.4) we obtain that they are identity on the boundary $\partial[-1,1]^{3}$. Moreover, the inverses are given by similar formula, e.g.

$$
\left(L_{z}\right)^{-1}(x, y, z)=\left(x, y, h_{s_{z}(x, y)}^{-1}(z)\right)
$$

and it is straightforward to verify that these maps are in fact bi-Lipschitz.

Let us denote by $\pi_{x}, \pi_{y}$ and $\pi_{z}$ the projections to the corresponding coordinates, e.g. $\pi_{x}(x, y, z)=x$. By $\pi_{x, y}, \pi_{x, z}, \pi_{y, z}$ we denote the projection to corresponding planes, e.g. $\pi_{x, y}(x, y, z)=(x, y)$. Our aim is to construct mappings $s_{z}, s_{y}, s_{x}$ such that the following is true.

First of all, $L_{z}$ takes the eight cubes $\tilde{Q}_{\boldsymbol{v}(1)}$ and shifts their $z$-coordinate to (6.3) as in Fig. 19. This can be clearly done using a suitable Lipschitz mapping $s_{z}$ which has constant value on each $\pi_{x y}\left(\tilde{Q}_{\boldsymbol{v}(1)}\right)$. Then $L_{y}$ takes the cubes $L_{z}\left(\tilde{Q}_{\boldsymbol{v}(1)}\right)$ and moves them in the $y$-direction so that it maps their centers (with $y$-coordinate $\frac{1}{2}$ or $-\frac{1}{2}$ ) to points with $y$-coordinate 0 , i.e.,

$$
\text { for every }(x, z) \in \pi_{x, z}\left(L_{z}\left(\tilde{Q}_{\boldsymbol{v}(1)}\right)\right) \text { we have } s_{y}(x, z)= \pm \frac{1}{2} \text {. }
$$

It is clear that we can select such a Lipschitz map $s_{y}$. In the end $L_{x}$ takes cubes $L_{y}\left(L_{z}\left(\tilde{Q}_{\boldsymbol{v}(1)}\right)\right)$ and moves them in the $x$-direction so that it maps their centers (with $x$-coordinate $\frac{1}{2}$ or $-\frac{1}{2}$ ) to points with $x$-coordinate 0 , i.e.,

$$
\text { for every }(y, z) \in \pi_{y, z}\left(L_{y}\left(L_{z}\left(\tilde{Q}_{\boldsymbol{v}(1)}\right)\right)\right) \text { we have } s_{x}(y, z)= \pm \frac{1}{2} \text {. }
$$

Again we can choose such a Lipschitz map $s_{x}$. At the end we have that the centers of the cubes $L\left(\tilde{Q}_{\boldsymbol{v}(1)}\right)$ have both $x$ and $y$ coordinates 0 and hence they are in the tower formation.

It remains to show that (6.1) holds for carefully chosen $s_{z}, s_{y}$ and $s_{x}$ as above. We do not give the exact formulas for $s_{z}, s_{y}$ and $s_{x}$ as it would be lengthy and nontransparent. Instead we explain on a series of pictures that we can achieve that the images of the red pipes from Fig. 18 do not intersect $\mathcal{S}_{\theta}$ from Fig. 17. Let us start with those red tubes that are "parallel" to $x$ and $y$ coordinates and connect $\tilde{Q}_{\boldsymbol{v}(1)}$ to $\partial[-1,1]^{3}$. By (6.2) the mapping $L_{p}$ is just the identity on them. We draw projection to $x y$-plane of images of these tubes under each mapping $L_{z}, L_{y}$ and then $L_{x}$. 

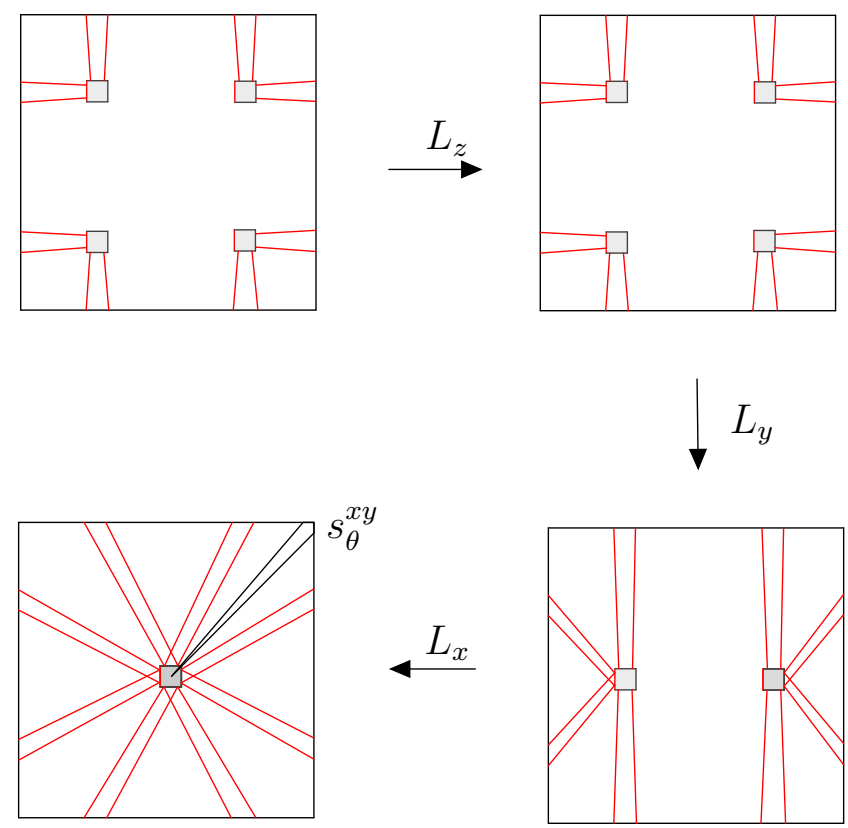

Fig. $21 L$ (red tubes parallel to $x$ and $y$ ) does not intersect $\mathcal{S}_{\theta}$ (projection to $x y$-plane)

The definition of corners (see Fig. 16) and $\mathcal{S}_{\theta}$ (see Fig. 17) guarantees that the projection of the red tubes ("parallel" to $x$ and $y$ coordinates) is really as in the last picture (bottom-left one) and hence it does not intersect $\mathcal{S}_{\theta}$ if $\theta$ is chosen sufficiently small (but fixed). Moreover, it is not difficult to see from these pictures that this can be done using Lipschitz functions $s_{z}, s_{y}$ and $s_{x}$ with the properties required above.

Now, let us consider the red tubes that are "parallel" to $x$ and $y$ axis and connect $\tilde{Q}_{\boldsymbol{v}(1)}$ together. By (6.2) the mapping $L_{p}$ is identity on them. Again we draw projections to $x y$-plane of images of these tubes under the mappings $L_{z}, L_{y}$ and then $L_{x}$ - see Fig. 22 below. The mapping $L_{z}$ just maps $\tilde{Q}_{\boldsymbol{v}(1)}$ to different $z$ coordinates but their $x y$-projection remains the same. Then $L_{y}$ maps the red tubes (originally parallel to $y$-axis) to some $\mathrm{S}$ shaped tubes (in the $y z$-projection) - the red object above and below the black cubes on the bottom-right picture. Then $L_{x}$ maps those $S$ shapes left to the black cube on the bottom-left picture. The red tubes parallel to $x$ are mapped below the black cubes by $L_{y}$ and then it remains below when we map by $L_{x}$. Again the image of the red tubes does not intersect $\mathcal{S}_{\theta}$ and it is not difficult to see from these pictures that this can be done using Lipschitz functions $s_{z}, s_{y}$ and $s_{x}$ with the properties required above. 

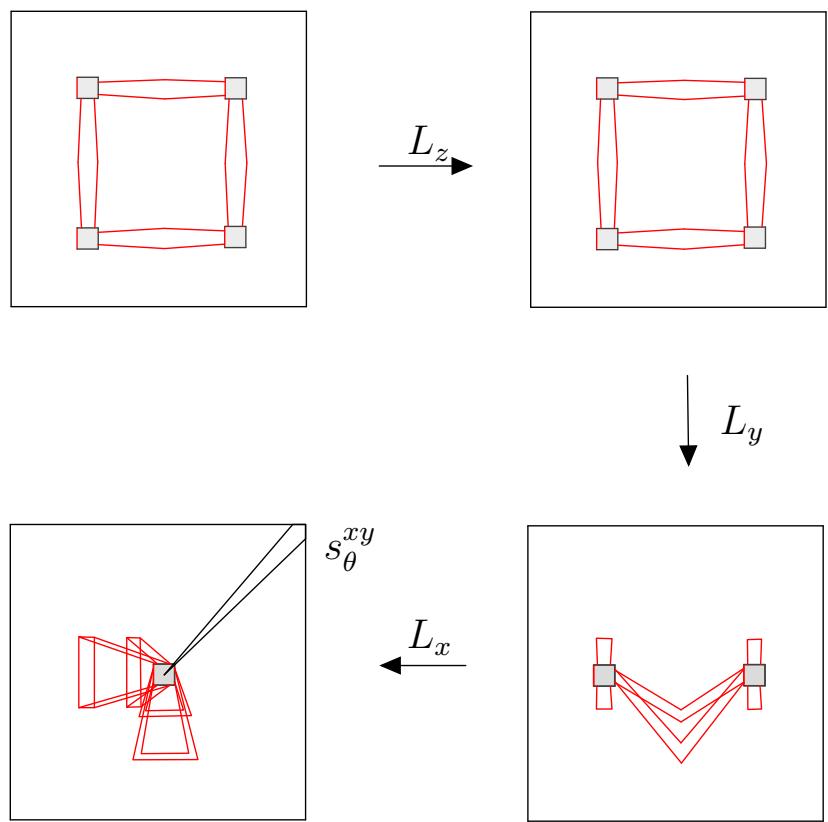

Fig. $22 L$ (red tubes parallel to $x$ and $y$ ) does not intersect $\mathcal{S}_{\theta}$ (projection to $x y$-plane)

It remains to consider the image of the red tubes that are "parallel" to $z$-axis. The situation is not as simple as in the previous case here, and we cannot simply use the mapping $L_{x} \circ L_{y} \circ L_{z}$. Therefore, we need to consider also a fourth mapping, which we denote by $L_{p}$. There are two reasons for this. First of all, the red tubes intersect $\partial[-1,1]^{3}$ and $L_{x} \circ L_{y} \circ L_{z}$ is the identity on $\partial[-1,1]^{3}$. Thus we have two squares from $\pi_{x, y}\left(\tilde{Q}_{\boldsymbol{v}(1)}\right) \times\{-1,1\}$ that intersect $\mathcal{S}_{\theta}$ (those in the upper-left corner, i.e. for $\boldsymbol{v}(1)=(1,1,1))$ and we have to move these two squares away using the mapping $L_{p}$. Secondly, $L_{x} \circ L_{y} \circ L_{z}$ is just a translation on $\partial \tilde{Q}_{\boldsymbol{v}(1)}$ but the red tubes intersect $\partial \tilde{Q}_{\boldsymbol{v}(1)}$. On the two faces of $\partial \tilde{Q}_{\boldsymbol{v}(1)}$ parallel to $x y$-plane we have two big red squares (intersection of red pipes and $\left.\partial \tilde{Q}_{\boldsymbol{v}(1)}\right)$ that are moved by $L_{x} \circ L_{y} \circ L_{z}$ so that the center of the image becomes $(0,0)$ in the $x y$-plane. Therefore these squares clearly intersect $\mathcal{S}_{\theta}$. We correct this by applying $L_{p}$ of the second generation (corresponding to the definition of $L_{2}$ ) and shift these red squares elsewhere.

Let us now explain how to define the mapping $L_{p}:[-1,1]^{3} \rightarrow[-1,1]^{3}$. Recall that $L_{p}$ is just an identity on a big part, see (6.2). Firstly, we define a mapping $\ell_{p}^{t}:[-1,1]^{2} \rightarrow[-1,1]^{2}$ for $t \in(0,1]$ that shrinks the red square $t$-times and moves it outside of the first quadrant (see Fig. 23) for small values of $t$. More precisely, let $0<a<1$ be a fixed number such that the red square is $[-1+a, 1-a]^{2}$. We define

$$
\ell_{p}^{t}(x)=t x-(1,1)+(t, t) \text { for } x \in[-1+a, 1-a]^{2} .
$$

It is clear that

$$
\ell_{p}^{t}\left([-1+a, 1-a]^{2}\right)=[-1+t a,-1+2 t-t a]^{2}
$$


and hence it moves the red square outside of the first quadrant for $t=\frac{1}{2}$. Moreover, we define $\ell_{p}^{t}$ on $[-1,1]^{2} \backslash[-1+a, 1-a]^{2}$ (similarly to $(2.4)$ ) so that the whole mapping is bi-Lipschitz (with constant $C \frac{1}{t}$ ) and it is identity on $\partial[-1,1]^{2}$.

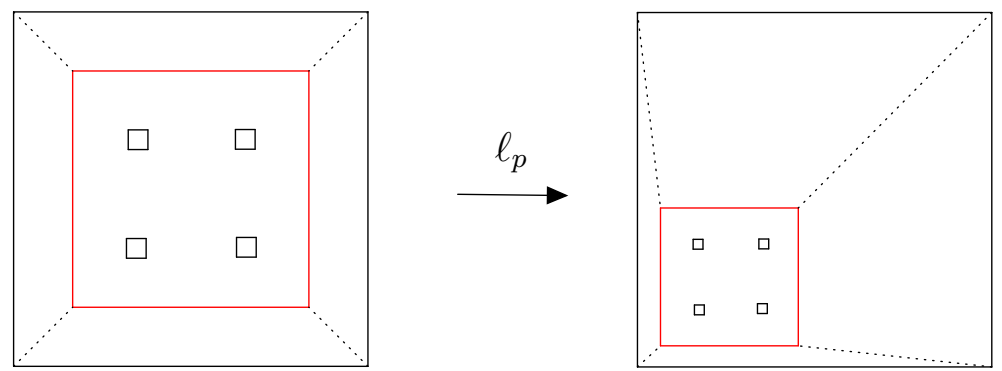

Fig. 23 Definition of the mapping $\ell_{p}^{t}$

We define

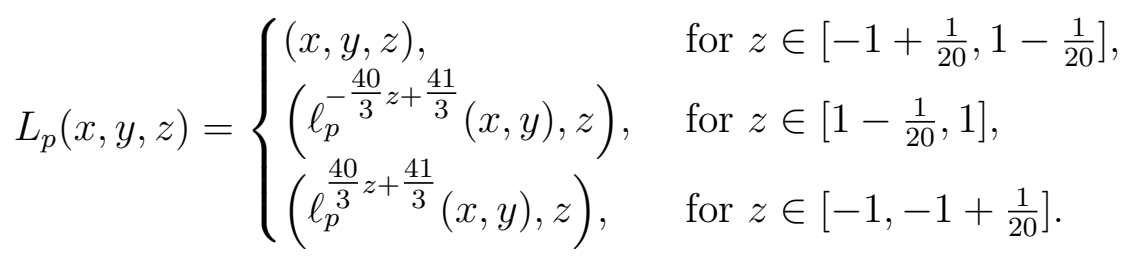

It is easy to see that this mapping is bi-Lipschitz and maps $[-1,1]^{3}$ onto $[-1,1]^{3}$. Moreover, it maps the red square on the planes $z=1$ and $z=-1$ outside of the first quadrant and it does a similar thing for nearby values of $z$. Now, we can define

$$
L_{1}:=L_{x} \circ L_{y} \circ L_{z} \circ L_{p} .
$$

Let us now briefly explain how to define $L_{2}$ and glue things together. Then we show the key property (6.1) for red tubes parallel to $z$-axis. We know that $L_{1}\left(\tilde{Q}_{\boldsymbol{v}(1)}\right)$ are cubes of side-length $2 \tilde{r}_{1}$. Let us denote their centers as $s_{\boldsymbol{v}(1)}$, i.e.,

$$
L_{1}\left(\tilde{Q}_{\boldsymbol{v}(1)}\right)=Q\left(s_{\boldsymbol{v}(1)}, \tilde{r}_{1}\right) .
$$

We define

$$
L_{z}^{2}(x)=s_{\boldsymbol{v}(1)}+\tilde{r}_{1} L_{z}\left(\frac{1}{\tilde{r}_{1}}\left(x-s_{\boldsymbol{v}(1)}\right)\right) \text { for } x \in Q\left(s_{\boldsymbol{v}(1)}, \tilde{r}_{1}\right) .
$$

It is easy to check that $L_{z}^{2}$ are bi-Lipschitz (with the same bi-Lipschitz constant as $\left.L_{z}\right)$ and they map $Q\left(s_{\boldsymbol{v}(1)}, \tilde{r}_{1}\right)$ onto $Q\left(s_{\boldsymbol{v}(1)}, \tilde{r}_{1}\right)$. In a similar spirit we define

$$
L_{p}^{2}(x)=s_{\boldsymbol{v}(1)}+\tilde{r}_{1} L_{p}\left(\frac{1}{\tilde{r}_{1}}\left(x-s_{\boldsymbol{v}(1)}\right)\right) \text { for } x \in Q\left(s_{\boldsymbol{v}(1)}, \tilde{r}_{1}\right)
$$

but we would like to define it also on $Q\left(s_{\boldsymbol{v}(1)}+2 \tilde{r}_{1} \mathbf{e}_{3}, \tilde{r}_{1}\right)$ and $Q\left(s_{\boldsymbol{v}(1)}-2 \tilde{r}_{1} \mathbf{e}_{3}, \tilde{r}_{1}\right)$ (here $\left.\mathbf{e}_{3}=(0,0,1)\right)$ so we connect it with the identity mapping outside of these three cubes. Formally we define (compare with (6.5))

$$
L_{p}(x, y, z)= \begin{cases}\left(\ell_{p}^{\frac{1}{3} z}(x, y), z\right), & \text { for }(x, y, z) \in Q((0,0,2), 1), \\ \left(\ell_{p}^{-\frac{1}{3} z}(x, y), z\right), & \text { for }(x, y, z) \in Q((0,0,-2), 1),\end{cases}
$$


and it is easy to see that $L_{p}$ is now identity on $\partial\left([-1,1]^{2} \times[-3,3]\right)$. The definition (6.6) now defines $L_{p}^{2}$ also on $Q\left(s_{\boldsymbol{v}(1)}+2 \tilde{r}_{1} \mathbf{e}_{3}, \tilde{r}_{1}\right)$ and $Q\left(s_{\boldsymbol{v}(1)}-2 \tilde{r}_{1} \mathbf{e}_{3}, \tilde{r}_{1}\right)$. It is not difficult to see that $L_{p}^{2}$ shifts the red squares on the $z$ boundaries of $\partial Q\left(s_{\boldsymbol{v}(1)}, \tilde{r}_{1}\right)$ "outside of the first quadrant" similarly as $L_{p}$ does for $[-1,1]^{3}$. Similarly we define $L_{y}^{2}$ (resp. $L_{x}^{2}$ ) on the cubes $L_{z}^{2}\left(L_{p}^{2}\left(Q\left(s_{\boldsymbol{v}(1)}, \tilde{r}_{1}\right)\right)\right)$ (respectively on $\left.L_{y}^{2}\left(L_{z}^{2}\left(L_{p}^{2}\left(Q\left(s_{\boldsymbol{v}(1)}, \tilde{r}_{1}\right)\right)\right)\right)\right)$.

Finally, we show that (6.1) holds for the red tubes from Fig. 18 parallel to $z$-axis for the mapping $L_{p}^{2} \circ L_{x} \circ L_{y} \circ L_{z} \circ L_{p}$. Again we show a series of pictures for the projections to $x y$-plane. The red squares correspond to intersection of the red pipes with $\partial[-1,1]^{2}$ (respectively $\tilde{Q}_{\boldsymbol{v}(1)}$ on the last two pictures).
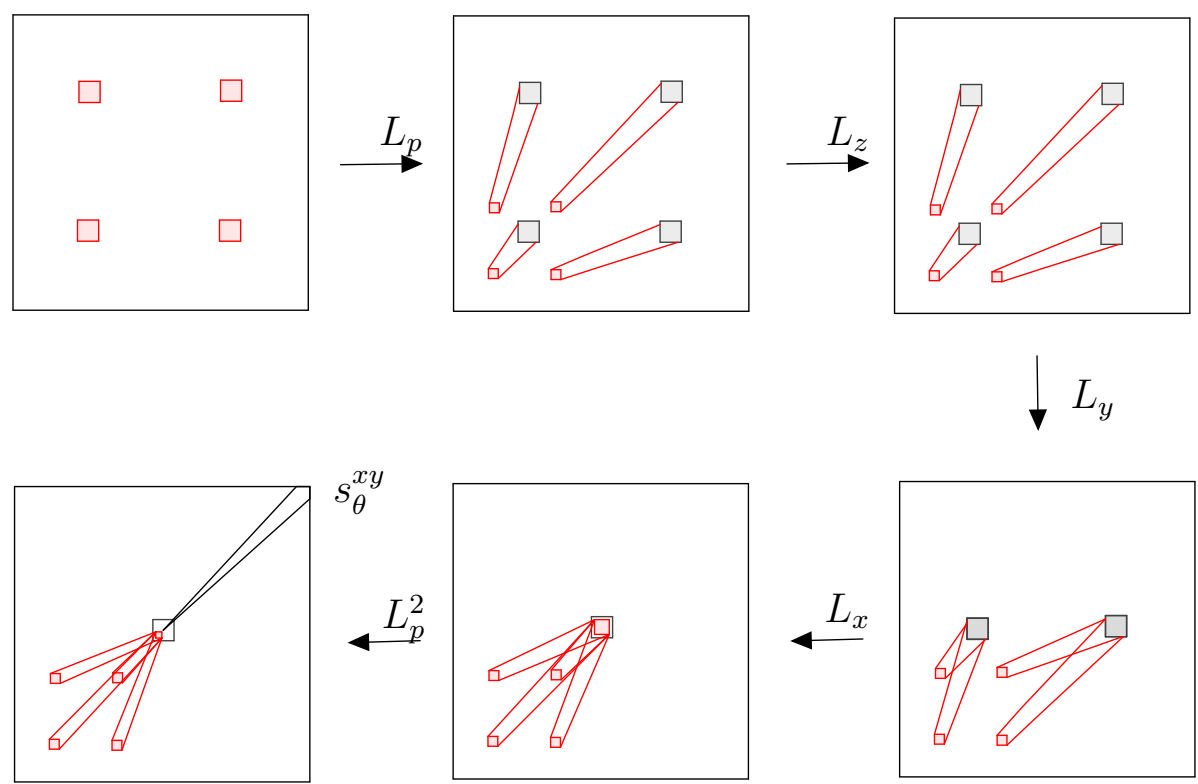

Fig. $24 L$ (red tubes parallel to $z$ ) does not intersect $\mathcal{S}_{\theta}$ (projection to $x y$-plane)

Our definition of $\mathcal{S}_{\theta}$ (see Fig. 17) guarantees that the projection of the red tubes ("parallel" to $z$-coordinate) does not intersect $\mathcal{S}_{\theta}$. Again it is not difficult to see from these pictures that this can be done using Lipschitz functions $s_{z}, s_{y}$ and $s_{x}$ with the properties required above. This finishes the proof of (6.1) and also the construction of $L_{1}$.

\section{Proof of TheOREM 1.3}

Proof of Theorem 1.3. Recall that the Cantor sets $C_{A}$ and $C_{B}$ are defined at the beginning of section 5 and that the set $\mathcal{S}_{\theta}$ is defined at the beginning of previous section. Suppose that:

(1) Mapping $g: \mathbb{R}^{3} \rightarrow \mathbb{R}^{3}$ is the homeomorphism (defined in section 5) which maps the Cantor set $C_{A}$ onto the Cantor set $C_{B}$ and maps each set $Q_{\boldsymbol{v}(k)}^{\prime} \backslash Q_{\boldsymbol{v}(k)}$ onto the corresponding set $\tilde{Q}_{\boldsymbol{v}(k)}^{\prime} \backslash \tilde{Q}_{\boldsymbol{v}(k)}$ radially and maps corners to corners.

(2) Mapping $L: \mathbb{R}^{3} \rightarrow \mathbb{R}^{3}$ is the bi-Lipschitz map (defined in section 6) which takes the Cantor set $C_{B}$ onto the Cantor's tower $C_{B}^{T}$ and for which the preimage $(L \circ g)^{-1}\left(\mathcal{S}_{\theta}\right)$ is contained in the union of the corners (see (6.1)). 
(3) Let $\omega_{\theta}$ be the mapping which is the identity on $\mathbb{R}^{3} \backslash \mathcal{S}_{\theta}$ and winds $\mathcal{S}_{\theta}$ in such a way that the angle $\theta$ is stretched to the angle $2 \pi+\theta$, so that the glued mapping is continuous.

We need to wind $\omega_{\theta}$ not radially but in supremum norm. Formally let $S: \mathbb{R}^{3} \rightarrow \mathbb{R}^{3}$ be a bi-Lipschitz mapping which maps the unit cube $(-1,1)^{3}$ onto a cylinder $B^{2}(0,1) \times(-1,1)$ naturally (so that $S\left(\mathcal{S}_{\theta}\right)$ corresponds to angle $\left.\varphi \in\left[\frac{\pi}{4}-\frac{\theta}{2}, \frac{\pi}{4}+\frac{\theta}{2}\right]\right)$. Let $(r, \varphi)$ be the polar coordinates of a point $\left(x_{1}, x_{2}\right)$ and define $\tilde{\omega}_{\theta}: \mathbb{R}^{3} \rightarrow \mathbb{R}^{3}$ as the winding map (the radial one)

$\tilde{\omega}_{\theta}(x)= \begin{cases}\left(r \cos \left(a(\theta, \varphi)+\frac{\pi}{4}-\frac{\theta}{2}\right), r \sin \left(a(\theta, \varphi)+\frac{\pi}{4}-\frac{\theta}{2}\right), x_{3}\right), & \text { if } x \in \mathcal{S}_{\theta} \\ x, & \text { otherwise, }\end{cases}$

where $a(\theta, \varphi):=\frac{\theta+2 \pi}{\theta}\left(\varphi-\frac{\pi}{4}+\frac{\theta}{2}\right)$. Then we set

$$
\omega_{\theta}=S^{-1} \circ \tilde{\omega}_{\theta} \circ S \text {. }
$$

Now we define the mapping $f:(-1,1)^{3} \rightarrow(-1,1)^{3}$ by

$$
f:=g^{-1} \circ L^{-1} \circ \omega_{\theta} \circ L \circ g .
$$

This composition maps $C_{A}$ onto $C_{B}$, then $C_{B}$ onto Cantor tower $C_{B}^{T} \subset(0,0) \times[-1,1]$, then winds twice around $(0,0) \times[-1,1]$ and then maps everything back to $C_{A}$. In this way $C_{A} \subset \mathcal{B}_{f}$ and $C_{A} \subset f\left(\mathcal{B}_{f}\right)$.

Step 1: First we need to show that $f$ is absolutely continuous on almost every line parallel to coordinate axis. Because $f$ is obviously a locally Lipschitz map outside the set $C_{A}$ (as it is a composition of locally Lipschitz maps there) it suffices to show that $f$ is absolutely continuous along those line segments $I$ parallel to coordinate axes for which $I \cap C_{A} \neq \emptyset$. For this purpose, suppose that $I_{i}$ is a line segment parallel to $x_{i}$-axis, such that $I_{i} \cap C_{A} \neq \emptyset$. We claim that actually

$$
f(x)=x \quad \text { for all } x \in I_{i} .
$$

To show this, we first recall the following definitions from section 6 :

$$
\mathbb{B} \mathbb{A D}:=\left\{x \in(-1,1)^{3}: L(g(x)) \in \mathcal{S}_{\theta}\right\}
$$

and

$$
\begin{aligned}
\mathbb{B A D}_{k} & :=\left\{x \in \bigcup_{\boldsymbol{v}(k) \in \mathbb{V}^{k}} Q_{\boldsymbol{v}(k)}^{\prime} \backslash Q_{\boldsymbol{v}(k)}: L(g(x)) \in \mathcal{S}_{\theta}\right\} \\
& =\left\{x \in \bigcup_{\boldsymbol{v}(k) \in \mathbb{V}^{k}} Q_{\boldsymbol{v}(k)}^{\prime} \backslash Q_{\boldsymbol{v}(k)}: x \in \mathbb{B} \mathbb{A D}\right\} .
\end{aligned}
$$

It follows from (6.1) in the construction of the bi-Lipschitz map $L$ that

$$
\mathbb{B A D}_{k} \subset \bigcup_{\boldsymbol{v}(k) \in \mathbb{V}^{k}} C_{\boldsymbol{v}(k)},
$$

and hence using (5.9) we have

$$
\mathcal{L}^{3}\left(\mathbb{B A D}_{k}\right) \lesssim 2^{-2 k}
$$


On the other hand, it follows from the definition of the sets $C_{\boldsymbol{v}(k)}$ that $I_{i} \cap C_{\boldsymbol{v}(k)}=\emptyset$ for every $\boldsymbol{v}(k)$ (since $I_{i} \cap C_{A} \neq \emptyset$ ) which implies

$$
I_{i} \cap \mathbb{B} \mathbb{A D}=\emptyset .
$$

Thus, for every $x \in I_{i}$ we have

$$
\begin{aligned}
f(x) & =\left(g^{-1} \circ L^{-1} \circ \omega_{\theta} \circ L \circ g\right)(x) \\
& =\left(g^{-1} \circ L^{-1} \circ \mathrm{id} \circ L \circ g\right)(x)=x,
\end{aligned}
$$

and the claim follows.

Step 2: Next we will show that

$$
\int_{(-1,1)^{3}}|D f(x)|^{2} \log ^{\alpha}(e+|D f(x)|) \mathrm{d} x<\infty
$$

for every $\alpha<-1$. For this purpose we observe first that $\omega_{\theta}(x)=x$ for all $x \in$ $(0,0) \times[-1,1]$, and that $L\left(g\left(C_{A}\right)\right) \subset(0,0) \times[-1,1]$. Then, as in (7.2), we obtain $f=\mathrm{id}$ on $C_{A}$. Therefore

$$
|D f(x)|=1 \quad \text { for almost every } x \in C_{A} .
$$

Thus, it suffices to estimate $|D f(x)|$ on $(-1,1)^{3} \backslash C_{A}$. For this purpose, we point out that

(i) $g$ is a locally bi-Lipschitz map on $(-1,1)^{3} \backslash C_{A}$ and

$$
g\left((-1,1)^{3} \backslash C_{A}\right)=(-1,1)^{3} \backslash C_{B} .
$$

(ii) $h:=L^{-1} \circ \omega_{\theta} \circ L$ is a Lipschitz map on $(-1,1)^{3} \backslash C_{B}$ and

$$
h\left((-1,1)^{3} \backslash C_{B}\right)=(-1,1)^{3} \backslash C_{B}
$$

and $h$ satisfies Lusin's $\left(N^{-1}\right)$ condition.

(iii) $g^{-1}$ is a locally Lipschitz map on $(-1,1)^{3} \backslash C_{B}$.

Analogously to the reasoning in the paragraph before (4.7) we obtain from (i)-(iii) that the chain rule applies to the mapping $f=g^{-1} \circ h \circ g$ almost everywhere outside the set $C_{A}$, and therefore

$$
\begin{aligned}
|D f(x)| & \leq\left|D g^{-1}(h(g(x)))\right| \cdot|D h(g(x))| \cdot|D g(x)| \\
& \leq \operatorname{Lip}(h)\left|D g^{-1}(h(g(x)))\right| \cdot|D g(x)|
\end{aligned}
$$

for almost every $x \in(-1,1)^{3} \backslash C_{A}$. Next we recall that $f=\mathrm{id}$ outside the set $\mathbb{B} \mathbb{A D}$ (see (7.2)) and therefore it suffices to show that

$$
\int_{\mathbb{B} \mathbb{A} D}|D f|^{2} \log ^{\alpha}(e+|D f|)=\sum_{k=1}^{\infty} \int_{\mathbb{B A D}_{k}}|D f|^{2} \log ^{\alpha}(e+|D f|)<\infty
$$

for every $\alpha<-1$. By (5.4) we have

$$
|D g(x)| \sim 2^{k-\beta k},
$$

for almost every $x \in \mathbb{B A D}_{k}$. Moreover, we also have

$$
(h \circ g)\left(Q_{\boldsymbol{v}(k)}^{\prime} \backslash Q_{\boldsymbol{v}(k)}\right)=\tilde{Q}_{\boldsymbol{v}(k)}^{\prime} \backslash \tilde{Q}_{\boldsymbol{v}(k)},
$$


and thus by (5.6)

$$
\left|D g^{-1}(g(h(x)))\right| \lesssim 2^{k \beta}
$$

for almost every $x \in \mathbb{B A D}_{k}$. If we now combine (7.1), (7.3), (7.4) and (7.5) we get

$$
\sum_{k=1}^{\infty} \int_{\mathbb{B A D}_{k}}|D f|^{2} \log ^{\alpha}(e+|D f|) \sim \sum_{k=0}^{\infty} 2^{-2 k} 2^{2 k} \log ^{\alpha}\left(e+2^{k}\right) \sim \sum_{k=1}^{\infty} k^{\alpha}<\infty
$$

for every $\alpha<-1$, and the claim follows. This is already enough to prove Theorem 1.3. However, it is interesting to know what level of integrability we may obtain for the distortion functions $K_{O}(\cdot, f)$ and $K_{I}(\cdot, f)$ in the construction. Therefore, we will add one additional step in the proof to estimate the distortions as well.

Step 3: Recall again that $f=\mathrm{id}$ on $(-1,1)^{3} \backslash \mathbb{B A D}$ and therefore

$$
\begin{array}{ll}
K_{I}(x, f)=1 & \text { for almost every } x \in(-1,1)^{3} \backslash \mathbb{B} \mathbb{A D}, \text { and } \\
K_{O}(x, f)=1 & \text { for almost every } x \in(-1,1)^{3} \backslash \mathbb{B} \mathbb{D} .
\end{array}
$$

As before we can apply the chain rule almost everywhere outside of $C_{A}$ and we get

$$
\begin{aligned}
K_{O}(x, f)=\frac{|D f(x)|^{n}}{J_{f}(x)} & \leq \frac{\left|D g^{-1}(h(g(x)))\right|^{n} \operatorname{Lip}^{n}(h)|D g(x)|^{n}}{J_{g^{-1}}(h(g(x))) J_{h}(g(x)) J_{g}(x)} \\
& \leq C K_{O}\left(h(g(x)), g^{-1}\right) K_{O}(x, g) .
\end{aligned}
$$

Analogously we obtain for adjugate matrices $|\operatorname{adj}(A B)| \leq \operatorname{adj}(A) \operatorname{adj}(B)$ and hence

$$
K_{I}(x, f) \leq C K_{I}\left(h(g(x)), g^{-1}\right) K_{I}(x, g) .
$$

From the estimates (5.5) and (5.7) in section 5.4 and from the fact $\mathbb{B A D}_{k} \subset G_{k} \cup E_{k}$ (see (6.1)) it follows that

$$
K_{I}(x, f) \leq C 2^{4 k} \quad \text { and } \quad K_{O}(x, f) \leq C 2^{3 k}
$$

for almost every $x \in \mathbb{B A D}_{k}$. Using this and (5.9) we get

$$
\begin{aligned}
\int_{(-1,1)^{3}} K_{I}(x, f)^{p} \mathrm{~d} x & =\int_{(-1,1)^{3} \backslash \mathbb{B A D}} K_{I}(x, f)^{p} \mathrm{~d} x+\sum_{k=1}^{\infty} \int_{\mathbb{B A D}_{k}} K_{I}(x, f)^{p} \mathrm{~d} x \\
& \leq 2^{3}+\sum_{k=1}^{\infty} 2^{-2 k} 2^{4 k p}<\infty
\end{aligned}
$$

for every $p<\frac{1}{2}$. Similarly, we get

$$
\begin{aligned}
\int_{(-1,1)^{3}} K_{O}(x, f)^{q} \mathrm{~d} x & =\int_{(-1,1)^{3} \backslash \mathbb{B} \mathbb{A D}} K_{O}(x, f)^{q} \mathrm{~d} x+\sum_{k=1}^{\infty} \int_{\mathbb{B A D}_{k}} K_{O}(x, f)^{q} \mathrm{~d} x \\
& \leq 2^{3}+\sum_{k=1}^{\infty} 2^{-2 k} 2^{3 k q}<\infty
\end{aligned}
$$

for every $q<\frac{2}{3}$. 
Remark 7.1. As it was pointed out in the introduction, we do not know what happens for the size of the branch set of continuous, discrete and open mappings with positive Jacobian almost everywhere in the borderline space $W^{1, n-1}$. However, it is possible to slightly improve the integrability of the distortions in Theorem 1.3 by modifying the construction of the mapping $f$ in the proof:

Proposition 7.2. Denote $Q_{0}:=(-1,1)^{3}$, and suppose that

$$
0<q<2 / 3 \text { and } 0<r<2 / 3 \text {. }
$$

Then there exists a continuous, discrete and open mapping of finite distortion $f \in$ $W^{1, p}\left(Q_{0}, Q_{0}\right)$, for all $p \in[1,2)$, with

$$
K_{I}(\cdot, f) \in L^{q}\left(Q_{0}\right) \quad \text { and } \quad K_{O}(\cdot, f) \in L^{r}\left(Q_{0}\right)
$$

such that $J_{f}>0$ almost everywhere and

$$
\mathcal{L}^{3}\left(\mathcal{B}_{f}\right)>0 \quad \text { and } \quad \mathcal{L}^{3}\left(f\left(\mathcal{B}_{f}\right)\right)>0 .
$$

We will only sketch the proof of the proposition above and highlight the differences with the construction in Theorem 1.3:

Sketch of the proof of Proposition 7.2. Instead of considering only two Cantor sets we will work now with three different Cantor sets

$$
C_{A}:=C\left[\left\{a_{k}\right\}_{k=1}^{\infty}\right], \quad C_{B}:=C\left[\left\{b_{k}\right\}_{k=1}^{\infty}\right] \quad \text { and } \quad C_{C}:=C\left[\left\{c_{k}\right\}_{k=1}^{\infty}\right]
$$

defined by the sequences

$$
a_{k}=\frac{1}{2}\left(1+\frac{1}{2^{k}}\right), \quad b_{k}=2^{-\beta k} \quad \text { and } \quad c_{k}=\frac{1}{2}\left(1+\frac{1}{2^{k \gamma}}\right),
$$

where $\beta>0$ can be chosen with a similar way as in the proof of Theorem 1.3, and the exponent $0<\gamma<1$ is defined later. In addition, suppose that the mappings $g, L$, and $\omega_{\theta}$ are defined similarly as in the proof of Theorem 1.3. We also consider a new homeomorphism $h:(-1,1)^{3} \rightarrow(-1,1)^{3}$ which maps the Cantor set $C_{C}$ onto $C_{B}$. The mapping $h$ can be defined with a similar fashion as the homeomorphism $g$ in section 5 . Then we set

$$
f:=h^{-1} \circ L^{-1} \circ \omega_{\theta} \circ L \circ g .
$$

One may check that this will give us a Sobolev mapping with positive Jacobian almost everywhere. Moreover, by calculating similarly as in the proof of Theorem 1.3 we get the following estimates:

(1) First we estimate the Sobolev norm in the set $\mathbb{B} \mathbb{A D}$ simply by applying the chain rule similarly as in the proof of Theorem 1.3. This gives us the following estimate:

$$
\int_{\mathbb{B A D}}|D f(x)|^{p} \mathrm{~d} x \lesssim \sum_{k=1}^{\infty} 2^{-2 k}\left(2^{k} \cdot 1\right)^{p} .
$$

The sum above converges for all $0<p<2$.

(2) To estimate the Sobolev norm of the mapping outside the set $\mathbb{B} \mathbb{A D}$ we observe by analyzing the map

$$
f=h^{-1} \circ \operatorname{id} \circ g
$$


in each of the sets $S_{k}$ more carefully (for the definition of the set $S_{k}$, see section 5) that

$$
D f(x) \sim\left(\begin{array}{ccc}
1 & 0 & 0 \\
0 & 1 & 0 \\
0 & 0 & 2^{\alpha k} 2^{-\gamma k}
\end{array}\right) \quad \text { for every } x \in S_{k}
$$

up to some permutation of the column vectors. This will give us the following estimate:

$$
\int_{(-1,1)^{3} \backslash \mathbb{B A D}}|D f(x)|^{p} \mathrm{~d} x \lesssim \sum_{k=1}^{\infty} 2^{-k}\left(2^{k} 2^{-\gamma k}\right)^{p} .
$$

The sum above converges whenever $\gamma>\frac{p-1}{p}$.

(3) We estimate the integral of $K_{I}^{q}$ in the set $\mathbb{B} \mathbb{A D}$ by applying the chain rule, which gives us:

$$
\int_{\mathbb{B A D}} K_{I}(x, f)^{q} \mathrm{~d} x \lesssim \sum_{k=1}^{\infty} 2^{-2 k}\left(2^{2 k} 2^{2 \gamma k}\right)^{q} .
$$

This sum converges whenever $0<\gamma<\frac{1-q}{q}$.

(4) Next, by applying the representation for the matrix $D f(x)$ in (2) we may estimate the integral of $K_{I}^{q}$ in the set $(-1,1)^{3} \backslash \mathbb{B} \mathbb{A D}$ as follows:

$$
\int_{(-1,1)^{3} \backslash \mathbb{B A D}} K_{I}(x, f)^{q} \mathrm{~d} x \lesssim \sum_{k=1}^{\infty} 2^{-k}\left(2^{2 k} 2^{-2 \gamma k}\right)^{q} .
$$

This sum converges whenever $\gamma>\frac{2 q-1}{2 q}$.

(5) We estimate the integral of $K_{O}^{r}$ in the set $\mathbb{B} \mathbb{A D}$ by simply applying the chain rule. This gives us the following estimate:

$$
\int_{\mathbb{B A D}} K_{O}(x, f)^{r} \mathrm{~d} x \lesssim \sum_{k=1}^{\infty} 2^{-2 k}\left(2^{2 k} 2^{2 \gamma k}\right)^{r} .
$$

This sum converges whenever $0<\gamma<\frac{1-r}{r}$.

(6) Again, by applying the representation of $D f(x)$ in (2), we may estimate the integral of $K_{O}^{r}$ in the set $(-1,1)^{3} \backslash \mathbb{B} \mathbb{A D}$ as follows:

$$
\int_{(-1,1)^{3} \backslash \mathbb{B} \mathbb{A D}} K_{O}(x, f)^{r} \mathrm{~d} x \lesssim \sum_{k=1}^{\infty} 2^{-k}\left(2^{k} 2^{-\gamma k}\right)^{r} .
$$

This sum converges whenever $\gamma>\frac{r-1}{r}$.

Suppose now that the parameters $1 \leq p<2,0<q<2 / 3$ and $0<r<2 / 3$ are fixed. Then we may choose the parameter $\gamma$ such a way that it satisfies the following condition

$$
\max \left\{\frac{p-1}{p}, \frac{r-1}{r}, \frac{2 q-1}{2 q}\right\}<\gamma<\min \left\{\frac{1-r}{r}, \frac{1-q}{q}\right\}
$$


More precisely, it is enough to choose $\gamma$ such that

$$
\frac{1}{2}<\gamma<\frac{1}{2}+\varepsilon
$$

where $\varepsilon>0$ is some sufficiently small number depending only on the values of the parameters $q$ and $r$. With such a parameter $\gamma$ we may see that the mapping $f$ satisfies all the estimates (1)-(6) above. Other parts of the proof we leave for the reader.

\section{Acknowledgements}

The authors want to express their gratitude to Kai Rajala, Aapo Kauranen and Pawel Goldstein for their valuable comments. The authors would also like to thank the anonymous referees for their very helpful comments that greatly improved the readability of the manuscript.

\section{REFERENCES}

1. M. Aaltonen, Monodromy representations of completed coverings, Rev. Mat. Iberoam. 32 (2016), no. 2, 533-570.

2. M. Aaltonen and P. Pankka, Local monodromy of branched covers and dimension of the branch set, Preprint.

3. L. Ambrosio, N. Fusco and D. Pallara, Functions of bounded variation and free discontinuity problems, Oxford Mathematical Monographs, The Clarendon Press, Oxford University Press, New York, 2000.

4. K. Astala, T. Iwaniec and G. Martin, Elliptic partial differential equations and quasiconformal mappings in the plane, Princeton University Press, Princeton, NJ, 2009.

5. J. Ball, Global invertibility of Sobolev functions and the interpenetration of matter, Proc. Roy. Soc. Edinburgh Sect. A 88 (1981), 315-328.

6. C.J. Bishop, Quasiconformal mappings which increase dimension, Acad. Sci. Fenn. Ser. A I Math. 24 (1999), 397-407.

7. A.V. Chernavskiı̌, Finite-to-one open mappings of manifolds (Russian), Mat. Sb. 65 (1964), 357-369.

8. A.V. Chernavskiı , Remarks on the paper "On finite-to-one open mappings of manifolds" (Russian), Mat. Sb. 66 (1965), 471-472.

9. M. Csörnyei, S. Hencl and J. Malý, Homeomorphisms in the Sobolev space $W^{1, n-1}$, J. Reine Angew. Math. 644 (2010), 221-235.

10. F.W. Gehring and J. Väisälä, The coefficients of quasiconformality of domains in space, Acta Math. 114 (1965), 1-70.

11. C.-Y. Guo, Mappings of finite distortion: global homeomorphism theorem, J. Geom. Anal. 25 (2015), 1969-1991.

12. J. Heinonen and P. Koskela, Sobolev mappings with integrable dilatations, Arch. Rational. Mech. Anal. 125 (1993), 81-97.

13. J. Heinonen and S. Rickman, Geometric branched covers between generalized manifolds, Duke Math. J., 113 (2002), no. 3, 465-529.

14. J. Heinonen and S. Rickman, Quasiregular maps $\mathbf{S}^{3} \rightarrow \mathbf{S}^{3}$ with wild branch sets, Topology, $\mathbf{3 7}$ (1998), no. 1, 1-24.

15. S. Hencl and P. Koskela, Lectures on mappings of finite distortion, Lecture Notes in Mathematics, Vol. 2096, 2014, XI, 176 p.

16. S. Hencl and K. Rajala, Optimal assumptions for discreteness, Arch. Ration. Mech. Anal 207 (2013), 775-783.

17. S. Hencl and V. Tengvall, Sharpness of the the differentiability almost everywhere and capacitary estimates for Sobolev mappings, Rev. Mat. Iberoam. 33 (2017), no. 2, 595-622. 
18. T. Iwaniec, P. Koskela and J. Onninen, Mappings of finite distortion: monotonicity and continuity, Invent. Math. 144 (2001), no. 3, 507-531.

19. T. Iwaniec and G. Martin, Geometric function theory and non-linear analysis, Oxford Mathematical Monographs. The Clarendon Press, Oxford University Press, New York, 2001.

20. T. Iwaniec and G. Martin, The Beltrami equation, Mem. Amer. Math. Soc. 191 (2008), no. 893.

21. T. Iwaniec and V. Šverák, On mappings with integrable dilatation, Proc. Amer. Math. Soc. 118 (1993), 181-188.

22. J. Kauhanen, P. Koskela and J. Malý, Mappings of finite distortion: discreteness and openness, Arch. Ration. Mech. Anal. 160 (2001), no. 2, 135-151.

23. J. Kauhanen, P. Koskela and J. Malý, Mappings of finite distortion: condition N, Michigan Math. J. 49 (2001), no. 1, 169-181.

24. J. Kauhanen, P. Koskela, J. Malý, J. Onninen and X. Zhong, Mappings of finite distortion: Sharp Orlicz-conditions, Rev. Mat. Iberoam. 19 (2003), 857-872.

25. P. Koskela and J. Malý, Mappings of finite distortion: the zero set of the Jacobian, J. Eur. Math. Soc. (JEMS) 5 (2003), no. 2, 95-105.

26. P. Koskela and J. Onninen, Mappings of finite distortion: capacity and modulus inequalities, J. Reine Angew. Math. 599 (2006), 1-26.

27. O. Martio, A capacity inequality for quasiregular mappings, Ann. Acad. Sci. Fenn. Ser. A I Math. 474 (1970), 1-18.

28. O. Martio, S. Rickman and J. Väisälä, Deffinitions for quasiregular mappings, Ann. Acad. Sci. Fenn. Ser. A I Math. 448 (1969), 1-40.

29. J. Onninen, Differentiability of monotone Sobolev functions, Real Anal. Exch. 26 (2000), no. 2, 761-772.

30. E. A. Poletskiǐ, The modulus method for non-homeomorphic quasiregular mappings (Russian), Mat. Sb. 83 (1970), 261-273.

31. Y. G. Reshetnyak, Space mappings with bounded distortion, Translations of Mathematical Monographs, 73. American Mathematical Society, Providence, RI, 1989.

32. S. Rickman, Quasiregular mappings. Ergebnisse der Mathematik und ihrer Grenzgebiete (3) [Results in Mathematics and Related Areas (3)], 26. Springer-Verlag, Berlin, 1993.

33. V. Šverák, Regularity properties of deformations with finite energy, Arch. Rational Mech. Anal. 100 (1988), 105-127.

34. V. Tengvall, Differentiability in the Sobolev space $W^{1, n-1}$, Calc. Var. Partial Differential Equations 51 (2014), no. 1-2, 381-399.

35. J. Väisälä, Discrete open mappings on manifolds, Ann. Acad. Sci. Fenn. Ser. A I Math. 392 (1966), 1-10.

36. J. Väisälä, Modulus and capacity inequalities for quasiregular mappings, Ann. Acad. Sci. Fenn. Ser. A I Math. 509 (1972), 1-14.

37. J. Väisälä, Two new characterizations for quasiconformality, Ann. Acad. Sci. Fenn. Ser. A I 362 (1965), 1-12.

(Chang-Yu Guo) Department of Mathematics, University of Fribourg, Fribourg, SWITZERLAND

E-mail address: changyu.guo@unifr.ch

(Stanislav Hencl) Department of Mathematical Analysis, Charles University, Sokolovská

83, 18600 Prague 8, Czech Republic

E-mail address: hencl@karlin.mff.cuni.cz

(Ville Tengvall) Department of Mathematics And Statistics, University of JyväSkylä, P.O. Box 35, FI-40014 Jyvëskylä, Finland

E-mail address: ville.tengvall@jyu.fi 